\title{
ON NONLINEAR SCHRÖDINGER EQUATIONS WITH ATTRACTIVE INVERSE-POWER POTENTIALS
}

\author{
VAN DUONG DINH
}

\begin{abstract}
We study the Cauchy problem for nonlinear Schrödinger equations with attractive inverse-power potentials. By using variational arguments, we first determine a sharp threshold of global well-posedness and blow-up for the equation in the mass-supercritical case. We next study the existence and orbital stability of standing waves for the problem in the mass-subcritical and mass-critical cases. In the mass-critical case, we give a detailed description of the blow-up behavior of standing waves when the mass tends to a critical value.
\end{abstract}

\section{IntRoduction AND MAIN RESULTS}

We consider the Cauchy problem for nonlinear Schrödinger equations with attractive inverse-power potentials

$$
\left\{\begin{aligned}
i \partial_{t} u+\Delta u+|x|^{-\sigma} u & = \pm|u|^{\alpha} u, \quad(t, x) \in \mathbb{R} \times \mathbb{R}^{d} \\
u(0) & =u_{0}
\end{aligned}\right.
$$

where $u: \mathbb{R} \times \mathbb{R}^{d} \rightarrow \mathbb{C}, u_{0}: \mathbb{R}^{d} \rightarrow \mathbb{C}, 0<\sigma<\min \{2, d\}$ and $\alpha>0$. The plus (resp. minus) sign in front of the nonlinearity corresponds to the defocusing (resp. focusing) case.

The Schrödinger equations with inverse-power potentials have attracted much attention recently. In the case of inverse-square potential $\sigma=2$, one has the following results: see Burq-Planchon-Stalker-TahvildarZadeh [6] for Strichartz estimates; Okazawa-Suzuki-Yokota [33] for the local and global well-posedness of the Cauchy problem; Zhang-Zheng [39] for the energy scattering in the defocusing case; Killip-Miao-Visan-ZhangZheng [25] for Sobolev spaces adapted to the Schrödinger operator with inverse-square potentials; KillipMurphy-Visan-Zheng [24], Zheng [40], Lu-Miao-Murphy [30] and Dinh [11] for the global existence, blowup and energy scattering in the focusing case; Killip-Miao-Visan-Zhang-Zheng [23] for the global existence and scattering in the energy-critical case; Csobo-Genoud [9] for the classification of minimial mass blow-up solutions and Bensouilah-Dinh-Zhu [4] for the stability and instability of standing waves. In the case of Coulomb potential $\sigma=1$, one has results of Benguria-Jeanneret [3] for the existence and uniqueness of positive solutions of semilinear elliptic equations; Chadam-Glassey [8], Hayashi-Ozawa [22] and Lions [28] for the global existence and time decay of global solutions for the Hartree equations and Miao-Zhang-Zheng [31] for the global existence, blow-up and energy scattering. In the case of slowly decaying potentials $0<\sigma<2$, we refer to Mizutani [32] for Strichartz estimates; Fukaya-Ohta [14] for the strong instability of standing waves; Guo-Wang-Yao [20] for the blow-up and energy scattering of the focusing 3D cubic NLS and Li-Zhao [27] for the orbital stability of standing waves.

This paper is a continuation of [13] where nonlinear Schrödinger equations with repulsive (i.e. the minus sign in front of $|x|^{-\sigma} u$ ) inverse-power potentials in the energy space were considered. The local well-posedness (LWP) for (1.1) in the energy space $H^{1}$ was studied in [13]. More precisely, the author showed that (1.1) is locally well-posed in $H^{1}$ for both energy-subcritical and energy-critical cases. Moreover, the time of existence depends only on the $H^{1}$-norm of initial data. In the energy-subcritical case, this LWP coincides with the usual local theory. In the energy-critical case, this LWP is stronger than the usual one in which the time of existence depends not only on the $H^{1}$-norm of initial data but also on its profile. The proof is based on the perturbation argument of Zhang in [38] by using Strichartz estimates in Lorentz spaces and viewing the potential as a sub-critical nonlinear term. A direct consequence of this local theory is the following global well-posedness result for (1.1).

Theorem 1.1 (Global existence [13]). Let $u_{0} \in H^{1}$. Suppose that

2010 Mathematics Subject Classification. 35Q44; 35Q55.

Key words and phrases. Nonlinear Schrödinger equation, Inverse-power potential, Standing waves, Stability, Global wellposedness, Blow-up. 
- in the defocusing case:

- (Energy-subcritical case) $0<\sigma<\min \{2, d\}$ and $0<\alpha<\frac{4}{d-2}$ if $d \geq 3(0<\alpha<\infty$ if $d=1,2)$;

- (Energy-critical case) $0<\sigma<2$ if $d \geq 4\left(0<\sigma<\frac{3}{2}\right.$ if $\left.d=3\right)$ and $\alpha=\frac{4}{d-2}$;

- in the focusing case:

- (Mass-subcritical case) $0<\sigma<\min \{2, d\}$ and $0<\alpha<\frac{4}{d}$;

- (Mass-critical case) $0<\sigma<\min \{2, d\}, \alpha=\frac{4}{d}$ and $\left\|u_{0}\right\|_{L^{2}}<\|Q\|_{L^{2}}$, where $Q$ is the unique (up to symmetries) positive radial solution to the elliptic equation

$$
\Delta Q-Q+|Q|^{\frac{4}{d}} Q=0 .
$$

Then there exists a unique global solution to (1.1). Moreover, the global solution u satisfies for $0<\sigma<2$ if $d \geq 4\left(0<\sigma<\frac{3}{2}\right.$ if $\left.d=3\right)$ and any compact interval $J \subset \mathbb{R}$,

$$
\sup _{(p, q) \in S}\|u\|_{L^{p}\left(J, W^{1, q}\right)} \leq C\left(\left\|u_{0}\right\|_{H^{1}},|J|\right),
$$

where $(p, q) \in S$ means that $(p, q)$ is a Schrödinger admissible pair.

1.1. Sharp threshold for global existence and blow-up. The first part of this paper is devoted to the global well-posedness for the focusing problem (1.1) in the mass-supercritical case. Before stating our results, let us introduce some notations. By a standing wave, we mean a solution to the focusing problem (1.1) of the form $e^{i \omega t} \phi(x)$, where $\omega \in \mathbb{R}$ is a frequency and $\phi \in H^{1}$ is a nontrivial solution to the elliptic equation

$$
-\Delta \phi+\omega \phi-|x|^{-\sigma} \phi-|\phi|^{\alpha} \phi=0 \text {. }
$$

It is well-known (see e.g. [17, Theorem 8.38]) that the minimizing problem

$$
\mu_{1}:=\inf \left\{\|\nabla v\|_{L^{2}}^{2}-G(v): v \in H^{1},\|v\|_{L^{2}}=1\right\}
$$

is attained by a positive function $\Phi$, where

$$
G(v):=\int|x|^{-\sigma}|v(x)|^{2} d x .
$$

Moreover, $\mu_{1}$ is the simple first eigenvalue corresponding to the eigenfunction $\Phi$ of the operator $-\Delta-|x|^{-\sigma}$. We also have from the Virial Theorem (see e.g. [10, Theorem 6.2.8]) that $\mu_{1}$ is negative. By the definition of $\mu_{1}$, we see that

$$
\mu_{1}\|v\|_{L^{2}}^{2} \leq\|\nabla v\|_{L^{2}}^{2}-G(v)
$$

for any $v \in H^{1}$. It is worth noticing that if $\omega \leq-\mu_{1}$, then the equation (1.3) does not admit positive solutions. In fact, suppose that $\phi$ is a positive solution of (1.3). By multiplying both sides of (1.3) with $\Phi$ and integrating by parts, we get

$$
\left(\omega+\mu_{1}\right) \int \phi(x) \Phi(x) d x=\int \phi^{\alpha+1}(x) \Phi(x)>0 .
$$

In the case $\omega>-\mu_{1}$, there exists at least one solution to (1.3) which is spherically symmetric and positive. To see this, we define the following action functional

$$
S_{\omega}(v):=E(v)+\frac{\omega}{2} M(v)=\frac{1}{2}\|\nabla v\|_{L^{2}}^{2}-\frac{1}{2} G(v)+\frac{\omega}{2}\|v\|_{L^{2}}^{2}-\frac{1}{\alpha+2}\|v\|_{L^{\alpha+2}}^{\alpha+2},
$$

and the corresponding Nehari functional

$$
K_{\omega}(v):=\left.\partial_{\lambda} S_{\omega}(\lambda v)\right|_{\lambda=1}=\|\nabla v\|_{L^{2}}^{2}-G(v)+\omega\|v\|_{L^{2}}^{2}-\|v\|_{L^{\alpha+2}}^{\alpha+2} .
$$

Note that the elliptic equation (1.3) can be written as $S_{\omega}^{\prime}(\phi)=0$. Consider the minimizing problem

$$
d(\omega):=\inf \left\{S_{\omega}(v): v \in H^{1} \backslash\{0\}, K_{\omega}(v)=0\right\},
$$

and define the set of all minimizers for (1.6) by

$$
\mathcal{M}_{\omega}:=\left\{v \in H^{1} \backslash\{0\}: K_{\omega}(v)=0, S_{\omega}(v)=d(\omega)\right\} .
$$

Proposition 1.2. Let $d \geq 1,0<\sigma<\min \{2, d\}$ and $0<\alpha<\frac{4}{d-2}$ if $d \geq 3(0<\alpha<\infty$ if $d=1,2)$. If $\omega>-\mu_{1}$, then $d(\omega)>0$ and $d(\omega)$ is attained by a function which is a solution to the elliptic equation (1.3). Moreover, every minimizer for $d(\omega)$ is of the form $e^{i \theta} \phi(x)$, where $\phi$ is a positive radially symmetric function. 
Notice that $\phi$ solves the ordinary differential equation

$$
\phi^{\prime \prime}(r)+\frac{d-1}{r} \phi^{\prime}(r)+\left(r^{-\sigma}-\omega\right) \phi(r)+\phi^{\alpha+1}(r)=0, \quad r \in(0,+\infty) .
$$

Using the general results of Shioji-Watanabe [35], we have (see Appendix) that for any $\omega>-\mu_{1}, 0<\sigma<1$, $d \geq 3$ and $0<\alpha<\frac{4}{d-2}$, there exists a unique positive solution to (1.7). A same argument has been used in [1] to show the uniqueness of positive ground states for the inhomogeneous Gross-Pitaevskii equation.

We now denote the set of nontrivial solutions of (1.3) by

$$
\mathcal{A}_{\omega}:=\left\{v \in H^{1} \backslash\{0\}: S_{\omega}^{\prime}(v)=0\right\} .
$$

Definition 1.3. A function $\phi \in \mathcal{A}_{\omega}$ is called a ground state for (1.3) if it minimizes $S_{\omega}$ over the set $\mathcal{A}_{\omega}$. The set of ground states for (1.3) is denoted by $\mathcal{G}_{\omega}$. In particular,

$$
\mathcal{G}_{\omega}=\left\{\phi \in \mathcal{A}_{\omega}: S_{\omega}(\phi) \leq S_{\omega}(v), \forall v \in \mathcal{A}_{\omega}\right\} .
$$

Proposition 1.4 (Existence of ground states [14]). Let $d \geq 1,0<\sigma<\min \{2, d\}$ and $0<\alpha<\frac{4}{d-2}$ if $d \geq 3$ $(0<\alpha<\infty$ if $d=1,2)$. If $\omega>-\mu_{1}$, then the set $\mathcal{G}_{\omega}$ is not empty, and it is characterized by

$$
\mathcal{G}_{\omega}=\left\{v \in H^{1} \backslash\{0\}: S_{\omega}(v)=d(\omega), K_{\omega}(v)=0\right\} .
$$

We now denote the functional

$$
Q(v):=\|\nabla v\|_{L^{2}}^{2}-\frac{\sigma}{2} G(v)-\frac{\beta}{\alpha+2}\|v\|_{L^{\alpha+2}}^{\alpha+2},
$$

where

$$
\beta:=\frac{d \alpha}{2}
$$

The functional $Q$ comes from the virial action

$$
\frac{d^{2}}{d t^{2}}\|x u(t)\|_{L^{2}}^{2}=8 Q(u(t))
$$

where $u$ is the solution to the focusing problem (1.1). Let $\phi \in \mathcal{G}_{\omega}$. We define the following sets

$$
\begin{aligned}
& \mathcal{K}_{\omega}^{-}:=\left\{v \in H^{1} \backslash\{0\}:\|v\|_{L^{2}} \leq\|\phi\|_{L^{2}}, S_{\omega}(v)<S_{\omega}(\phi), K_{\omega}(v)<0, Q(v)<0\right\}, \\
& \mathcal{K}_{\omega}^{+}:=\left\{v \in H^{1} \backslash\{0\}:\|v\|_{L^{2}} \leq\|\phi\|_{L^{2}}, S_{\omega}(v)<S_{\omega}(\phi), K_{\omega}(v)<0, Q(v)>0\right\} .
\end{aligned}
$$

We will see in Remark 3.3 and Lemma 3.7 that for $\omega$ large enough,

$$
\left\{v \in H^{1} \backslash\{0\}:\|v\|_{L^{2}} \leq\|\phi\|_{L^{2}}, S_{\omega}(v)<S_{\omega}(\phi), K_{\omega}(v)<0, Q(v)=0\right\}=\emptyset,
$$

hence

$$
\mathcal{K}_{\omega}^{-} \cup \mathcal{K}_{\omega}^{+}=\left\{v \in H^{1} \backslash\{0\}:\|v\|_{L^{2}} \leq\|\phi\|_{L^{2}}, S_{\omega}(v)<S_{\omega}(\phi), K_{\omega}(v)<0\right\} .
$$

We are now able to state our first result concerning the sharp threshold of global existence and blow-up for the focusing problem (1.1) in the mass-supercritical and energy-subcritical case.

Theorem 1.5. Let $d \geq 1,0<\sigma<\min \{2, d\}$ and $\frac{4}{d}<\alpha<\frac{4}{d-2}$ if $d \geq 3\left(\frac{4}{d}<\alpha<\infty\right.$ if $\left.d=1,2\right)$. Then there exists $\omega_{0}>-\mu_{1}$ such that for any $\omega \geq \omega_{0}$ and $\phi \in \mathcal{G}_{\omega}$, the following properties hold:

- If $u_{0} \in \mathcal{K}_{\omega}^{-}$and $|x| u_{0} \in L^{2}$, then the corresponding solution to (1.1) blows up in finite time.

- If $u_{0} \in \mathcal{K}_{\omega}^{+}$, then the corresponding solution to (1.1) exists globally in time.

The proof of finite time blow-up given in Theorem 1.5 is based on the variational argument of [14]. The key point (see Proposition 3.2) is to show for $\omega>-\mu_{1}$ large enough and $v \in H^{1} \backslash\{0\}$ satisfying

$$
\|v\|_{L^{2}} \leq\|\phi\|_{L^{2}}, \quad K_{\omega}(v) \leq 0, \quad Q(v) \leq 0
$$

it holds that

$$
Q(v) \leq 2\left(S_{\omega}(v)-S_{\omega}(\phi)\right) .
$$

The finite time blow-up then follows from (1.9), (1.11) and a classical convexity argument of Glassey [16]. We refer the reader to Section 3 for more details. 
1.2. Existence and stability of standing waves. The second part of this paper is devoted to the existence and stability of standing waves for the focusing problem (1.1) in the mass-subcritical and mass-critical cases. Given $a>0$, we consider the minimizing problem

$$
I(a):=\inf \left\{E(v): v \in H^{1},\|v\|_{L^{2}}^{2}=a\right\},
$$

where

$$
E(v):=\frac{1}{2}\|\nabla v\|_{L^{2}}^{2}-\frac{1}{2} G(v)-\frac{1}{\alpha+2}\|v\|_{L^{\alpha+2}}^{\alpha+2} .
$$

We denote the set of minimizers for $I(a)$ by

$$
\mathcal{N}(a):=\left\{v \in H^{1}: E(v)=I(a),\|v\|_{L^{2}}^{2}=a\right\} .
$$

By the Lagrange multiplier theorem, for each $v \in \mathcal{N}(a)$, there exists $\omega \in \mathbb{R}$ such that (1.3) holds with $v$ in place of $\phi$. In this case, $e^{i \omega t} v(x)$ is a solution to (1.1) with initial data $v$. One usually calls $e^{i \omega t} v$ the orbit of $v$. Moreover, if $v \in \mathcal{N}(a)$, i.e. $v$ is a minimizer for $I(a)$, then $e^{i \omega t} v$ is also a minimizer for $I(a)$ or $e^{i \omega t} v \in \mathcal{N}(a)$. We are also interested in the orbital stability for $\mathcal{N}(a)$ under the flow of the focusing problem (1.1).

Definition 1.6. The set $\mathcal{N}(a)$ is called orbitally stable under the flow of the focusing problem (1.1) if for every $\varepsilon>0$, there exists $\delta>0$ such that for any initial data $u_{0} \in H^{1}$ satisfying

$$
\inf _{v \in \mathcal{N}(a)}\left\|u_{0}-v\right\|_{H^{1}}<\delta
$$

the corresponding solution $u$ to (1.1) satisfies

$$
\inf _{v \in \mathcal{N}(a)}\|u(t)-v\|_{H^{1}}<\varepsilon
$$

for all $t \in \mathbb{R}$.

Note that the above definition of orbital stability implicitly requires that (1.1) has a unique global solution at least for initial data $u_{0}$ sufficiently close to $\mathcal{N}(a)$.

Remark 1.7. In the case of no potential and focusing mass-subcritical nonlinearity (i.e. $d \alpha<4$ ), by using the scaling technique, we can show that each $v \in \mathcal{N}(a)$ is actually a ground state for

$$
-\Delta \psi+\omega \psi-|\psi|^{\alpha} \psi=0
$$

where $\omega$ is the Lagrange multiplier, that is, $v$ minimizes the action functional $S_{\omega}$ over all solutions of (1.13). In fact, we will show that

$$
S_{\omega}(v) \leq S_{\omega}(\psi)
$$

for any solution $\psi$ of (1.13), where

$$
S_{\omega}(v)=\frac{1}{2}\|\nabla v\|_{L^{2}}^{2}+\frac{\omega}{2}\|v\|_{L^{2}}^{2}-\frac{1}{\alpha+2}\|v\|_{L^{\alpha+2}}^{\alpha+2} .
$$

Assume by contradiction that there exists a solution $\psi$ to $(1.13)$ such that $S_{\omega}(\psi)<S_{\omega}(v)$. Since $\psi$ is a solution of (1.13), we have the following Pohozaev identities

$$
\|\nabla \psi\|_{L^{2}}^{2}+\omega\|\psi\|_{L^{2}}^{2}-\|\psi\|_{L^{\alpha+2}}^{\alpha+2}=0, \quad \frac{2-d}{2}\|\nabla \psi\|_{L^{2}}^{2}-\frac{d \omega}{2}\|\psi\|_{L^{2}}^{2}+\frac{d}{\alpha+2}\|\psi\|_{L^{\alpha+2}}^{\alpha+2}=0 .
$$

Of course, similar identities hold for $v$ as well. From these identities, we infer that

$$
\|\psi\|_{L^{\alpha+2}}^{\alpha+2}=\frac{2(\alpha+2)}{\alpha} S_{\omega}(\psi), \quad\|\nabla \psi\|_{L^{2}}^{2}=d S_{\omega}(\psi), \quad \omega\|\psi\|_{L^{2}}^{2}=\frac{4-(d-2) \alpha}{\alpha} S_{\omega}(\psi),
$$

and $E(\psi)=\frac{(d \alpha-4) \omega}{2(4-(d-2) \alpha)}\|\psi\|_{L^{2}}^{2}$. Now set

$$
\lambda:=\left(\frac{a}{\|\psi\|_{L^{2}}^{2}}\right)^{\frac{\alpha}{4-d \alpha}}
$$

and define

$$
\psi_{\lambda}(x):=\lambda^{\frac{2}{\alpha}} \psi(\lambda x)
$$

We see that

$$
\left\|\psi_{\lambda}\right\|_{L^{2}}^{2}=\lambda^{\frac{4-d \alpha}{\alpha}}\|\psi\|_{L^{2}}^{2}=a
$$


Since $v \in \mathcal{N}(a)$, we have

$$
\frac{(d \alpha-4) \omega}{2(4-(d-2) \alpha)} a=E(v) \leq E\left(\psi_{\lambda}\right)=\lambda^{\frac{4-(d-2) \alpha}{\alpha}} E(\psi)=\lambda^{\frac{4-(d-2) \alpha}{\alpha}} \frac{(d \alpha-4) \omega}{2(4-(d-2) \alpha)}\|\psi\|_{L^{2}}^{2} .
$$

Since $d \alpha<4$, it follows that $a \geq \lambda^{\frac{4-(d-2) \alpha}{\alpha}}\|\psi\|_{L^{2}}^{2}$, hence

On the other hand,

$$
\lambda^{\frac{4-(d-2) \alpha}{\alpha}} \leq \frac{a}{\|\psi\|_{L^{2}}^{2}}=\lambda^{\frac{4-d \alpha}{\alpha}} \text { or } \lambda \leq 1 .
$$

$$
\frac{\alpha}{4-(d-2) \alpha} \omega\|\psi\|_{L^{2}}^{2}=S_{\omega}(\psi)<S_{\omega}(v)=\frac{\alpha}{4-(d-2) \alpha} \omega a,
$$

hence $\|\psi\|_{L^{2}}^{2}<a$ or $\lambda>1$ which is a contradiction. Thus, one gets (1.14) and the claim follows. In the presence of inverse-power potential, there is no scaling invariance for (1.1), so it is not clear whether or not each $v \in \mathcal{N}(a)$ is a ground state for (1.3).

Recently, Li-Zhao [27] studied the existence of standing waves and the orbital stability for $\mathcal{N}(a)$ in the mass-subcritical and mass-critical cases. Their proof is based on the concentration-compactness principle of P. L. Lions [29]. Our purpose here is to give a direct simple proof for the result of [27].

In the mass-subcritical case, i.e. $\alpha<\frac{4}{d}$, the energy functional is bounded from below on

$$
S(a):=\left\{v \in H^{1}:\|v\|_{L^{2}}^{2}=a\right\} .
$$

Thus for every $a>0$, we can find the global minimizer of the energy functional on $S(a)$. More precisely, we have the following result.

Theorem 1.8. Let $d \geq 1,0<\sigma<\min \{2, d\}, 0<\alpha<\frac{4}{d}$ and $a>0$. Then, it holds that:

- The set $\mathcal{N}(a)$ is not empty.

- If $v \in \mathcal{N}(a)$, then there exists a positive radially symmetric function $\phi \in H^{1}$ such that $v(x)=e^{i \theta} \phi(x)$ for some $\theta \in \mathbb{R}$.

- The set $\mathcal{N}(a)$ is orbitally stable under the flow of the focusing problem (1.1).

In the mass-critical case, i.e. $\alpha=\frac{4}{d}$, under an appropriate assumption on $a$, the energy functional is bounded from below on $S(a)$. We have the following existence and stability of standing waves.

Theorem 1.9. Let $d \geq 1,0<\sigma<\min \{2, d\}, \alpha=\frac{4}{d}$ and $0<a<a^{*}:=\|Q\|_{L^{2}}^{2}$, where $Q$ is the unique (up to symmetries) positive radial solution to (1.2). Then, it holds that:

- The set $\mathcal{N}(a)$ is not empty.

- If $v \in \mathcal{N}(a)$, then there exists a positive radially symmetric function $\phi \in H^{1}$ such that $v(x)=e^{i \theta} \phi(x)$ for some $\theta \in \mathbb{R}$.

- The set $\mathcal{N}(a)$ is orbitally stable under the flow of the focusing problem (1.1).

The proofs of Theorems 1.8 and 1.9 are based on variational arguments using the radial compactness embedding. If we denote

$$
H_{\mathrm{r}}^{1}:=\left\{v \in H^{1}: v \text { is radially symmetric }\right\},
$$

then it is well-known that the embedding $H_{\mathrm{r}}^{1} \hookrightarrow L^{q}$ is compact for any $2<q<\frac{2 d}{d-2}$ if $d \geq 3(2<q<\infty$ if $d=2$ ). Note that this compact embedding only holds in dimensions $d \geq 2$. The reason is that the inequality

$$
|v(x)| \leq C|x|^{\frac{1-d}{2}}\|v\|_{H^{1}}, \quad v \in H_{\mathrm{r}}^{1}
$$

gives no decay in the case $d=1$. However, if $v$ is in addition radially decreasing, then it holds (see e.g. [7, Appendix]) that

$$
|v(x)| \leq\left(\frac{d}{\left|\mathbb{S}^{d-1}\right|}\right)^{\frac{1}{2}}|x|^{-\frac{d}{2}}\|v\|_{L^{2}} .
$$

The above inequality yields the compact embedding

$$
H_{\mathrm{rd}}^{1} \hookrightarrow L^{q}, \quad 2<q<\frac{2 d}{d-2} \text { if } d \geq 3(2<q<\infty \text { if } d=1,2),
$$

where

$$
H_{\mathrm{rd}}^{1}:=\left\{v \in H_{\mathrm{r}}^{1}: v \text { is radially decreasing }\right\}
$$


For the reader's convenience, we give the proof of (1.16) in the Appendix.

1.3. Blow-up behavior of standing waves. We next study the blow-up behavior of standing waves as the mass tends to a critical value in the mass-critical case. To our knowledge, the first paper addressed the blow-up behavior of standing waves in the mass-critical case belongs to Guo-Seiringer [18]. They studied the behavior of minimizers for

$$
\min \left\{E_{b}(v): v \in H^{1},\|v\|_{L^{2}}=1\right\},
$$

where

$$
E_{b}(v):=\int_{\mathbb{R}^{2}}|\nabla v(x)|^{2} d x+\int_{\mathbb{R}^{2}} V(x)|v(x)|^{2} d x-\frac{b}{2} \int_{\mathbb{R}^{2}}|v(x)|^{4} d x
$$

and $V$ is a trapping potential which has finite isolated minima. This result has been extended to ring-shaped trapping potentials in [19], to periodic potentials in [36] and to attractive potential vanishing at infinity in [34]. In this paper, we have the following result.

Theorem 1.10. Let $d \geq 1,0<\sigma<\min \{2, d\}, \alpha=\frac{4}{d}$ and $a^{*}=\|Q\|_{L^{2}}^{2}$, where $Q$ is the unique (up to symmetries) positive radial solution to (1.2). Then, it holds that:

- If $a \geq a^{*}$, then there is no minimizer for $I(a)$.

- If $v_{a}$ is a non-negative minimizer for $I(a)$ with $0<a<a^{*}$, then $v_{a}$ blows up as a $\nearrow a^{*}$ in the sense that

$$
\lim _{a \nearrow a^{*}}\left\|\nabla v_{a}\right\|_{L^{2}}=\infty
$$

Moreover,

$$
\beta_{a}^{\frac{d}{2(2-\sigma)}} v_{a}\left(\beta_{a}^{\frac{1}{2-\sigma}} \cdot\right) \rightarrow \lambda_{0}^{\frac{d}{2}} Q\left(\lambda_{0} \cdot\right) \text { strongly in } H^{1} \text { as a } \nearrow a^{*}
$$

where

$$
\beta_{a}:=1-\left(\frac{a}{a^{*}}\right)^{\frac{2}{d}}, \quad \lambda_{0}:=\left(\frac{\sigma G\left(Q_{0}\right)}{d}\right)^{\frac{1}{2-\sigma}}, \quad Q_{0}=\frac{Q}{\|Q\|_{L^{2}}} .
$$

Note that since $\|\nabla|v|\|_{L^{2}} \leq\|\nabla v\|_{L^{2}}$, we can always assume that minimizers for $I(a)$ are non-negative. The proof is inspired by recent arguments of Phan [34] as follows. The first step is to derive energy estimates for $I(a)$ (see (5.2)). Using these estimates and a suitable change of variables, we show that the sequence of minimizers converges strongly in $H^{1}$ to an optimizer for the Gagliardo-Nirenberg (GN) inequality

$$
\|v\|_{L^{\frac{4}{d}+2}}^{\frac{4}{d}+2} \leq \frac{d+2}{d}\left(\frac{\|v\|_{L^{2}}}{\|Q\|_{L^{2}}}\right)^{\frac{4}{d}}\|\nabla v\|_{L^{2}}^{2} .
$$

It then follows from the uniqueness (up to symmetries) of optimizers for the GN inequality that the limit equals to $Q$ modulo symmetries. Finally, we determine the exact limit by matching the energy.

In the mass-supercritical case, i.e. $\alpha>\frac{4}{d}$, the energy functional is no longer bounded from below on $S(a)$. Indeed, let $v \in H^{1}$ be such that $\|v\|_{L^{2}}^{2}=a$. We define $v^{\lambda}(x):=\lambda^{\frac{d}{2}} v(\lambda x)$. It is clear that $\left\|v^{\lambda}\right\|_{L^{2}}^{2}=\|v\|_{L^{2}}^{2}=a$ and

$$
E\left(v^{\lambda}\right)=\frac{\lambda^{2}}{2}\|\nabla v\|_{L^{2}}^{2}-\frac{\lambda^{\sigma}}{2} G(v)-\frac{\lambda^{\beta}}{\alpha+2}\|v\|_{L^{\alpha+2}}^{\alpha+2},
$$

where $\beta$ is as in (1.8). Since $\alpha>\frac{4}{d}$ or $\beta>2$, it follows that $E\left(v^{\lambda}\right) \rightarrow-\infty$ as $\lambda \rightarrow+\infty$. There is thus no minimizer for $I(a)$ in this case. Although there is no minimizers for $I(a)$, one may find normalized solutions for (1.3) in the mass-supercritical case by following a recent method of Bellazzini-Boussaid-Jeanjean-Visciglia [2]. The idea is to consider the local minimizing problem

$$
\inf \{E(v): v \in S(a) \cap B(r)\},
$$

where

$$
B(r):=\left\{v \in H^{1}: H_{0}(v):=\|\nabla v\|_{L^{2}}^{2}-G(v) \leq r\right\} .
$$

This method works well for potentials $V$ satisfying

$$
\inf \left\{\|\nabla v\|_{L^{2}}^{2}+\int V|v|^{2} d x:\|v\|_{L^{2}}^{2}=1\right\}>0,
$$


for instance, $V=|x|^{2}$ or $V=\sum_{j=1}^{k} x_{j}^{2}$, where $x=\left(x_{1}, \cdots, x_{k}, \cdots, x_{d}\right)$. In the case of attractive inverse-power potential, the minimum of the spectrum is negative, and the method of [2] is not directly applicable.

After the paper is submitted, the author was informed by Prof. Ohta that the blow-up result given in Theorem 1.5 is actually proved by Fukaya-Ohta in [14]. In fact, they proved that if $u_{0} \in \mathcal{B}_{\omega}$ and $|x| u_{0} \in L^{2}$, where

$$
\mathcal{B}_{\omega}:=\left\{v \in H^{1}:\|v\|_{L^{2}} \leq\|\phi\|_{L^{2}}, S_{\omega}(v)<S_{\omega}(\phi),\|v\|_{L^{\alpha+2}}>\|\phi\|_{L^{\alpha+2}}, Q(v)<0\right\},
$$

then the corresponding solution to the focusing problem (1.1) blows up in finite time. Moreover, it is not hard to check that $\mathcal{K}_{\omega}^{-}=\mathcal{B}_{\omega}$.

This paper is organized as follows. In Section 2, we prove the existence of ground states given in Proposition 1.2. In Section 3, we give the proof of sharp threshold of global existence and blow-up for the focusing problem (1.1) given in Theorem 1.5. Section 4 is devoted to the existence and stability of standing waves given in Theorems 1.8 and 1.9. In Section 5, we study the blow-up behavior of standing waves in the mass-critical case. Finally, the uniqueness of positive radial solutions to (1.3) is given in Appendix.

\section{EXISTENCE OF GROUND STATES}

In this section, we prove the existence of ground states given in Proposition 1.2. To do so, we define the functional

$$
H_{\omega}(v):=\|\nabla v\|_{L^{2}}^{2}-G(v)+\omega\|v\|_{L^{2}}^{2} .
$$

Thanks to (1.5) and Hardy's inequality, we see that for $\omega>-\mu_{1}$ fixed,

$$
H_{\omega}(v) \sim\|v\|_{H^{1}}^{2} .
$$

More precisely, there exists $C>0$ such that

$$
\frac{\min \left\{C, \omega+\mu_{1}\right\}}{2}\|v\|_{H^{1}}^{2} \leq H_{\omega}(v) \leq \max \{1, \omega\}\|v\|_{H^{1}}^{2} .
$$

In fact, the upper bound follows easily from the fact $G(v) \geq 0$. To see the lower bound, we first have from (1.5) that

$$
H_{\omega}(v) \geq\left(\omega+\mu_{1}\right)\|v\|_{L^{2}}^{2} .
$$

On the other hand, by Hardy's inequality (see e.g. [39, Lemma 2.6])

$$
\int|x|^{-\sigma}|v(x)|^{2} d x \leq C\left\||\nabla|^{\sigma / 2} v\right\|_{L^{2}}^{2} \leq C\|\nabla v\|_{L^{2}}^{\sigma}\|v\|_{L^{2}}^{2-\sigma}
$$

and the fact $0<\sigma<2$, the Young inequality implies that

$$
G(v) \leq \frac{\sigma}{2}\|\nabla v\|_{L^{2}}^{2}+C\|v\|_{L^{2}}^{2}
$$

for some constant $C>0$. It follows that

$$
H_{\omega}(v) \geq \frac{2-\sigma}{2}\|\nabla v\|_{L^{2}}^{2}+(\omega-C)\|v\|_{L^{2}}^{2}
$$

By choosing $\lambda$ such that $\omega-C+\lambda\left(\omega+\mu_{1}\right) \geq 0$, we infer from (2.2) and (2.4) that

$$
(1+\lambda) H_{\omega}(v) \geq \frac{2-\sigma}{2}\|\nabla v\|_{L^{2}}^{2}+\left(\omega-C+\lambda\left(\omega+\mu_{1}\right)\right)\|v\|_{L^{2}}^{2}
$$

hence

$$
H_{\omega}(v) \geq \frac{2-\sigma}{2(1+\lambda)}\|\nabla v\|_{L^{2}}^{2} .
$$

This together with (2.2) imply

$$
2 H_{\omega}(v) \geq \frac{2-\sigma}{2(1+\lambda)}\|\nabla v\|_{L^{2}}^{2}+\left(\omega+\mu_{1}\right)\|v\|_{L^{2}}^{2}
$$

which shows the lower bound.

Note that the action functional can be rewritten as

$$
S_{\omega}(v)=\frac{1}{2} K_{\omega}(v)+\frac{\alpha}{2(\alpha+2)}\|v\|_{L^{\alpha+2}}^{\alpha+2}=\frac{1}{\alpha+2} K_{\omega}(v)+\frac{\alpha}{2(\alpha+2)} H_{\omega}(v) .
$$


Lemma 2.1. Let $d \geq 1,0<\sigma<\min \{2, d\}$ and $0<\alpha<\frac{4}{d-2}$ if $d \geq 3(0<\alpha<\infty$ if $d=1,2)$. If $\omega>-\mu_{1}$, then there exists $v \in H^{1} \backslash\{0\}$ such that $K_{\omega}(v)=0$. In particular, the minimizing problem (1.6) is well-defined.

Proof. Let $v \in H^{1} \backslash\{0\}$. If $K_{\omega}(v)=0$, we are done. If $K_{\omega}(v) \neq 0$, then for any $\lambda>0$,

$$
K_{\omega}(\lambda v)=\lambda^{2} H_{\omega}(v)-\lambda^{\alpha+2}\|v\|_{L^{\alpha+2}}^{\alpha+2} .
$$

Note that since $\omega>-\mu_{1}$, by $(1.5), H_{\omega}(v) \geq\left(\omega+\mu_{1}\right)\|v\|_{L^{2}}^{2}>0$. It follows that $K_{\omega}\left(\lambda_{0} v\right)=0$, where

$$
\lambda_{0}=\left(\frac{H_{\omega}(v)}{\|v\|_{L^{\alpha+2}}^{\alpha+2}}\right)^{\frac{1}{\alpha}}>0
$$

It closes the proof.

Lemma 2.2. $d(\omega)>0$.

Proof. Let $v \in H^{1} \backslash\{0\}$ be such that $K_{\omega}(v)=0$. Using (2.1) and the fact $H_{\omega}(v)=\|v\|_{L^{\alpha+2}}^{\alpha+2}$, the Sobolev embedding implies

$$
\|v\|_{L^{\alpha+2}}^{2} \leq C_{1}\|v\|_{H^{1}}^{2} \leq C_{2} H_{\omega}(v)=C_{2}\|v\|_{L^{\alpha+2}}^{\alpha+2}
$$

for some constants $C_{1}, C_{2}>0$. It follows that

$$
S_{\omega}(v)=\frac{\alpha}{2(\alpha+2)}\|v\|_{L^{\alpha+2}}^{\alpha+2} \geq \frac{\alpha}{2(\alpha+2)}\left(\frac{1}{C_{2}}\right)^{\frac{\alpha+2}{\alpha}} .
$$

The result follows by taking the infimum over $v \in H^{1} \backslash\{0\}$ with $K_{\omega}(v)=0$.

We denote the set of all minimizers for (1.6) by

$$
\mathcal{M}_{\omega}:=\left\{v \in H^{1} \backslash\{0\}: K_{\omega}(v)=0, S_{\omega}(v)=d(\omega)\right\} .
$$

It is well-known (see e.g. [14,15]) that if $\mathcal{M}_{\omega}$ is non-empty, then $\mathcal{M}_{\omega} \equiv \mathcal{G}_{\omega}$. In [14], Fukaya-Ohta makes use of the weak continuity of the potential energy (see e.g. [26, Theorem 11.4]) to show the non-emptiness of $\mathcal{M}_{\omega}$. In the following result, we give an alternative proof of this result.

Lemma 2.3. The set $\mathcal{M}_{\omega}$ is non-empty.

Proof. We first observe from Lemma 2.1 that if $v \in H^{1} \backslash\{0\}$ satisfying $K_{\omega}(v) \leq 0$, then there exists $\lambda_{0} \in(0,1]$ such that $K_{\omega}\left(\lambda_{0} v\right)=0$.

We next claim that any minimizing sequence for $d(\omega)$ can be chosen to be radially symmetric and radially decreasing. Indeed, let $\left(v_{n}\right)_{n \geq 1}$ be a minimizing sequence for $d(\omega)$. Let $v_{n}^{*}$ be the symmetric rearrangement of $v_{n}$. Note that the symmetric rearrangement preserves the $L^{p}$-norm and by Polya-Szego's inequality, $\left\|\nabla v_{n}^{*}\right\|_{L^{2}} \leq\left\|\nabla v_{n}\right\|_{L^{2}}$. We also have from the Hardy-Littlewood's inequality that

$$
\int|x|^{-\sigma}\left|v_{n}(x)\right|^{2} d x \leq \int|x|^{-\sigma}\left|v_{n}^{*}(x)\right|^{2} d x .
$$

It follows that $H_{\omega}\left(v_{n}^{*}\right) \leq H_{\omega}\left(v_{n}\right)$ and $K_{\omega}\left(v_{n}^{*}\right) \leq K_{\omega}\left(v_{n}\right)=0$. By the above observation, there exists $\left(\mu_{n}\right)_{n \geq 1} \subset(0,1]$ such that $K_{\omega}\left(\mu_{n} v_{n}^{*}\right)=0$ for all $n \geq 1$. We have

$$
S_{\omega}\left(\mu_{n} v_{n}^{*}\right)=\frac{\alpha}{2(\alpha+2)} \mu_{n}^{2} H_{\omega}\left(v_{n}^{*}\right) \leq \frac{\alpha}{2(\alpha+2)} H_{\omega}\left(v_{n}^{*}\right) \leq \frac{\alpha}{2(\alpha+2)} H_{\omega}\left(v_{n}\right)=S_{\omega}\left(v_{n}\right) .
$$

This shows that $\left(\mu_{n} v_{n}^{*}\right)_{n \geq 1}$ is also a minimizing sequence for $d(\omega)$.

We next show that any minimizing sequence for $d(\omega)$ is bounded in $H^{1}$. In fact, let $\left(v_{n}\right)_{n \geq 1}$ be a minimizing sequence for $d(\omega)$. It follows that $H_{\omega}\left(v_{n}\right)=\left\|v_{n}\right\|_{L^{\alpha+2}}^{\alpha+2}$ for all $n \geq 1$. By (2.5), we have

$$
S_{\omega}\left(v_{n}\right)=\frac{\alpha}{2(\alpha+2)} H_{\omega}\left(v_{n}\right)=\frac{\alpha}{2(\alpha+2)}\left\|v_{n}\right\|_{L^{\alpha+2}}^{\alpha+2} \rightarrow d(\omega)
$$

as $n \rightarrow \infty$. We infer that there exists $C>0$ such that

$$
H_{\omega}\left(v_{n}\right) \leq \frac{2(\alpha+2)}{\alpha} d(\omega)+C
$$

for all $n \geq 1$. Thanks to (2.1), we see that $\left(v_{n}\right)_{n \geq 1}$ is a bounded sequence in $H^{1}$. 
Now let $\left(v_{n}\right)_{n \geq 1}$ be a radially symmetric and radially decreasing minimizing sequence for $d(\omega)$. Since $\left(v_{n}\right)_{n \geq 1}$ is bounded in $H^{1}$, the compact embedding (1.16) implies that there exist $v \in H^{1}$ and a subsequence still denoted by $\left(v_{n}\right)_{n \geq 1}$ such that $v_{n} \rightarrow v$ weakly in $H^{1}$ and $v_{n} \rightarrow v$ strongly in $L^{q}$ for any $2<q<\frac{2 d}{d-2}$ if $d \geq 3(2<q<\infty$ if $d=1,2)$. This implies in particular that $v \neq 0$. Indeed, since $K_{\omega}\left(v_{n}\right)=0$ for all $n \geq 1$, by the same argument as in Lemma 2.2, there exists $C>0$ such that $\left\|v_{n}\right\|_{L^{\alpha+2}} \geq C$. By the strong convergence, we get $\|v\|_{L^{\alpha+2}} \geq C>0$.

We next claim that $G\left(v_{n}\right) \rightarrow G(v)$ as $n \rightarrow \infty$. To see this, we estimate

$$
\begin{aligned}
\left|G\left(v_{n}\right)-G(v)\right| & \leq c \int|x|^{-\sigma}|| v_{n}(x)|-| v(x)||\left(\left|v_{n}\right|+|v(x)|\right) d x \\
& \leq c \int|x|^{-\sigma}\left|v_{n}(x)-v(x)\right|\left(\left|v_{n}(x)\right|+|v(x)|\right) d x \\
& =c\left[\int_{B(0,1)}+\int_{B^{c}(0,1)}\right]|x|^{-\sigma}\left|v_{n}(x)-v(x)\right|\left(\left|v_{n}(x)\right|+|v(x)|\right) d x=: I_{1}+I_{2},
\end{aligned}
$$

where $B(0,1)$ the unit ball in $\mathbb{R}^{d}$ and $B^{c}(0,1)$ is its complement.

On $B(0,1)$, we have

$$
I_{1} \lesssim\left\||x|^{-\sigma}\right\|_{L^{\gamma}(B(0,1))}\left\|\left|v_{n}-v\right|\left(\left|v_{n}\right|+|v|\right)\right\|_{L^{\mu}} \lesssim\left\|v_{n}-v\right\|_{L^{\delta}}\left(\left\|v_{n}\right\|_{L^{\tau}}+\|v\|_{L^{\tau}}\right),
$$

where $\gamma, \mu, \delta, \tau \geq 1$ satisfy

$$
1=\frac{1}{\gamma}+\frac{1}{\mu}, \quad \frac{d}{\gamma}>\sigma, \quad \frac{1}{\mu}=\frac{1}{\delta}+\frac{1}{\tau}
$$

Here the second condition ensures that $\left\||x|^{-\sigma}\right\|_{L^{\gamma}(B(0,1))}<\infty$. Using the fact $v_{n} \rightarrow v$ for $2<q<\frac{2 d}{d-2}$ if $d \geq 3(2<q<\infty$ if $d=1,2)$ and the Sobolev embedding $H^{1} \subset L^{q}$, we see that if we are able to choose $2<\delta, \tau<\frac{2 d}{d-2}$ in the case $d \geq 3(2<\delta, \tau<\infty$ in the case $d=1,2)$ so that

$$
\frac{1}{\delta}+\frac{1}{\tau}<\frac{d-\sigma}{d}
$$

then $I_{1} \rightarrow 0$ as $n \rightarrow \infty$. The condition (2.6) is fulfilled if we take $\delta=\tau=\frac{2 d}{d-2}-\varepsilon$ with $0<\varepsilon<\frac{2 d(2-\sigma)}{(d-2)(d-\sigma)}$ in the case $d \geq 3$ and $\delta=\tau$ large enough in the case $d=1,2$.

On $B^{c}(0,1)$, we estimate

$$
I_{2} \lesssim\left\||x|^{-\sigma}\right\|_{L^{\gamma}\left(B^{c}(0,1)\right)}\left\|\left|v_{n}-v\right|\left(\left|v_{n}\right|+|v|\right)\right\|_{L^{\mu}} \lesssim\left\|v_{n}-v\right\|_{L^{\delta}}\left(\left\|v_{n}\right\|_{L^{\tau}}+\|v\|_{L^{\tau}}\right),
$$

where $\gamma, \mu, \delta, \tau \geq 1$ satisfy

$$
1=\frac{1}{\gamma}+\frac{1}{\mu}, \quad \frac{d}{\gamma}<\sigma, \quad \frac{1}{\mu}=\frac{1}{\delta}+\frac{1}{\tau}
$$

If we choose $2<\delta, \tau<\frac{2 d}{d-2}$ in the case $d \geq 3(2<\delta, \tau<\infty$ in the case $d=1,2)$ so that

$$
\frac{1}{\delta}+\frac{1}{\tau}>\frac{d-\sigma}{d}
$$

then $I_{2} \rightarrow 0$ as $n \rightarrow \infty$. The above condition is satisfied for $\delta=\tau=2+\varepsilon$ with $0<\varepsilon<\frac{2 \sigma}{d-\sigma}$.

Combining the above two cases, we prove that $G\left(v_{n}\right) \rightarrow G(v)$ as $n \rightarrow \infty$. It follows that

$$
K_{\omega}(v) \leq \liminf _{n \rightarrow \infty} K_{\omega}\left(v_{n}\right)=0 .
$$

There thus exists $\lambda_{0} \in(0,1]$ such that $K_{\omega}\left(\lambda_{0} v\right)=0$. By the definition of $d(\omega)$,

$$
d(\omega) \leq S_{\omega}\left(\lambda_{0} v\right)=\frac{\alpha}{2(\alpha+2)} \lambda_{0}^{2} H_{\omega}(v) \leq \frac{\alpha}{2(\alpha+2)} \liminf _{n \rightarrow \infty} H_{\omega}\left(v_{n}\right)=\liminf _{n \rightarrow \infty} S_{\omega}\left(v_{n}\right)=d(\omega) .
$$

This implies that $S_{\omega}\left(\lambda_{0} v\right)=d(\omega)$ or $\lambda_{0} v$ is a minimizer for $d(\omega)$. Moreover, all inequalities above are in fact equalities, that is, $\lambda_{0}=1, K_{\omega}(v)=0$ and $H_{\omega}(v)=\lim _{n \rightarrow \infty} H_{\omega}\left(v_{n}\right)$ which implies by (2.1) that $v_{n} \rightarrow v$ strongly in $H^{1}$. The proof is complete. 


\section{SHARP THRESHOLD FOR GLOBAL EXISTENCE AND BLOW-UP}

In this section, we prove the sharp threshold of global existence and blow-up for the focusing problem (1.1). To this end, we set

$$
v^{\lambda}(x):=\lambda^{\frac{d}{2}} v(\lambda x), \quad \lambda>0
$$

A direct computation shows

$$
\begin{aligned}
\left\|v^{\lambda}\right\|_{L^{2}}^{2} & =\|v\|_{L^{2}}^{2}, \quad\left\|\nabla v^{\lambda}\right\|_{L^{2}}^{2}=\lambda^{2}\|\nabla v\|_{L^{2}}^{2}, \\
G\left(v^{\lambda}\right) & =\lambda^{\sigma} G(v), \quad\left\|v^{\lambda}\right\|_{L^{\alpha+2}}^{\alpha+2}=\lambda^{\beta}\|v\|_{L^{\alpha+2}}^{\alpha+2},
\end{aligned}
$$

where $\beta$ is given in (1.8). It follows that

$$
S_{\omega}\left(v^{\lambda}\right)=\frac{\lambda^{2}}{2}\|\nabla v\|_{L^{2}}^{2}-\frac{\lambda^{\sigma}}{2} G(v)+\frac{\omega}{2}\|v\|_{L^{2}}^{2}-\frac{\lambda^{\beta}}{\alpha+2}\|v\|_{L^{\alpha+2}}^{\alpha+2}
$$

and

$$
Q\left(v^{\lambda}\right)=\lambda^{2}\|\nabla v\|_{L^{2}}^{2}-\frac{\sigma}{2} \lambda^{\sigma} G(v)-\frac{\beta}{\alpha+2} \lambda^{\beta}\|v\|_{L^{\alpha+2}}^{\alpha+2}=\lambda \partial_{\lambda} S_{\omega}\left(v^{\lambda}\right) .
$$

In particular, $Q(v)=\left.\partial_{\lambda} S_{\omega}\left(v^{\lambda}\right)\right|_{\lambda=1}$.

Lemma 3.1. Let $d \geq 1,0<\sigma<\min \{2, d\}, 0<\alpha<\frac{4}{d-2}$ if $d \geq 3(0<\alpha<\infty$ if $d=1,2)$ and $\omega>-\mu_{1}$. Let $\phi \in H^{1} \backslash\{0\}$ be a solution to (1.3). Then it holds that

$$
\begin{gathered}
\|\nabla \phi\|_{L^{2}}^{2}+\omega\|\phi\|_{L^{2}}^{2}-G(\phi)-\|\phi\|_{L^{\alpha+2}}^{\alpha+2}=0 \\
\frac{2-d}{2}\|\nabla \phi\|_{L^{2}}^{2}-\frac{d \omega}{2}\|\phi\|_{L^{2}}^{2}+\frac{(d-\sigma)}{2} G(\phi)+\frac{d}{\alpha+2}\|\phi\|_{L^{\alpha+2}}^{\alpha+2}=0 .
\end{gathered}
$$

In particular, $K_{\omega}(\phi)=Q(\phi)=0$.

Proof. By multiplying both sides of (1.3) with $\bar{\phi}$ and integrating over $\mathbb{R}^{d}$, we get the first identity in (3.5) which is $K_{\omega}(\phi)=0$. Multiplying both sides of (1.3) with $x \cdot \nabla \bar{\phi}$, integrating over $\mathbb{R}^{d}$ and taking the real part, we obtain the second identity in (3.5). Note that we only make formal computations here. Due to the singularity of the inverse-power potential at zero, we need to integrate on the annulus $\left\{x \in \mathbb{R}^{d}: r \leq|x| \leq R\right\}$ for $R>r>0$ and then take the limit as $R \rightarrow+\infty$ and $r \rightarrow 0$. We refer the reader to [12, Lemma 3.2] for detailed computations in the case of inverse-square potential. Multiplying both sides of the first identity with $\frac{d}{2}$ and adding to the second identity, we obtain $Q(v)=0$. The proof is complete.

Proposition 3.2. Let $d \geq 1,0<\sigma<\min \{2, d\}, \frac{4}{d}<\alpha<\frac{4}{d-2}$ if $d \geq 3\left(\frac{4}{d}<\alpha<\infty\right.$ if $\left.d=1,2\right)$ and $\omega>-\mu_{1}$. Let $\phi \in \mathcal{G}_{\omega}$ be such that $\left.\partial_{\lambda}^{2} S_{\omega}\left(\phi^{\lambda}\right)\right|_{\lambda=1} \leq 0$, where $\phi^{\lambda}$ is as in (3.1). Let $v \in H^{1} \backslash\{0\}$ be such that

$$
\|v\|_{L^{2}} \leq\|\phi\|_{L^{2}}, \quad K_{\omega}(v) \leq 0, \quad Q(v) \leq 0
$$

Then it holds that

$$
Q(v) \leq 2\left(S_{\omega}(v)-S_{\omega}(\phi)\right)
$$

Remark 3.3. It is easy to see from Proposition 3.2 that for $\phi \in \mathcal{G}_{\omega}$ satisfying $\left.\partial_{\lambda}^{2} S_{\omega}\left(\phi^{\lambda}\right)\right|_{\lambda=1} \leq 0$,

$$
\left\{v \in H^{1} \backslash\{0\}:\|v\|_{L^{2}} \leq\|\phi\|_{L^{2}}, S_{\omega}(v)<S_{\omega}(\phi), K_{\omega}(v)<0, Q(v)=0\right\}=\emptyset,
$$

Indeed, if there exists $v \in H^{1} \backslash\{0\}$ satisfying $\|v\|_{L^{2}} \leq\|\phi\|_{L^{2}}, S_{\omega}(v)<S_{\omega}(\phi), K_{\omega}(v)<0$ and $Q(v)=0$, then by Proposition 3.2,

which is a contradiction.

$$
0=Q(v) \leq 2\left(S_{\omega}(v)-S_{\omega}(\phi)\right)<0
$$

Proof of Proposition 3.2. The proof is similar to [14, Lemma 3.2], where $K_{\omega}(v) \leq 0$ is replaced by $\|v\|_{L^{\alpha+2}} \geq$ $\|\phi\|_{L^{\alpha+2}}$. For the reader's convenience, we give some details. If $K_{\omega}(v)=0$, then by Proposition 1.4 and $Q(v) \leq 0$, we have $S_{\omega}(\phi) \leq S_{\omega}(v) \leq S_{\omega}(v)-\frac{1}{2} Q(v)$. Suppose that $K_{\omega}(v)<0$. Let $v^{\lambda}$ be as in $(3.1)$ and define

$$
f(\lambda):=S_{\omega}\left(v^{\lambda}\right)-\frac{\lambda^{2}}{2} Q(v)=\frac{1}{2}\left(\frac{\sigma}{2} \lambda^{2}-\lambda^{\sigma}\right) G(v)+\frac{1}{\alpha+2}\left(\frac{\beta}{2} \lambda^{2}-\lambda^{\beta}\right)\|v\|_{L^{\alpha+2}}^{\alpha+2}+\frac{\omega}{2}\|v\|_{L^{2}}^{2} .
$$


We have

$$
K_{\omega}\left(v^{\lambda}\right):=\lambda^{2}\|\nabla v\|_{L^{2}}^{2}-\lambda^{\sigma} G(v)+\omega\|v\|_{L^{2}}^{2}-\lambda^{\beta}\|v\|_{L^{\alpha+2}}^{\alpha+2} .
$$

Since $\lim _{\lambda \rightarrow 0} K_{\omega}\left(v^{\lambda}\right)=\omega\|v\|_{L^{2}}^{2}>0$ and $K_{\omega}(v)<0$, it follows that there exists $\lambda_{0} \in(0,1)$ such that $K_{\omega}\left(v^{\lambda_{0}}\right)=0$. If we have $f\left(\lambda_{0}\right) \leq f(1)$, then it follows from $Q(v) \leq 0$ that

$$
S_{\omega}(\phi) \leq S_{\omega}\left(v^{\lambda_{0}}\right) \leq S_{\omega}\left(v^{\lambda_{0}}\right)-\frac{\lambda_{0}^{2}}{2} Q(v) \leq S_{\omega}(v)-\frac{1}{2} Q(v) .
$$

It remains to prove $f\left(\lambda_{0}\right) \leq f(1)$ which is in turn equivalent to

$$
G(v) \leq \frac{2\left(2 \lambda_{0}^{\beta}-\beta \lambda_{0}^{2}-2+\beta\right)}{(\alpha+2)\left(\sigma \lambda_{0}^{2}-2 \lambda_{0}^{\sigma}-\sigma+2\right)}\|v\|_{L^{\alpha+2}}^{\alpha+2} .
$$

Note that by (3.3), the condition $\left.\partial_{\lambda}^{2} S_{\omega}\left(\phi^{\lambda}\right)\right|_{\lambda=1} \leq 0$ is equivalent to

$$
\|\nabla \phi\|_{L^{2}}^{2}-\frac{\sigma(\sigma-1)}{2} G(\phi)-\frac{\beta}{\alpha+2}(\beta-1)\|\phi\|_{L^{\alpha+2}}^{\alpha+2} \leq 0 .
$$

Since $K_{\omega}(\phi)=Q(\phi)=0$, we have from $\sigma K_{\omega}(\phi)-(\sigma+1) Q(\phi)=0$ and (3.8) that

$$
\begin{aligned}
\omega \sigma\|\phi\|_{L^{2}}^{2} & =\|\nabla \phi\|_{L^{2}}^{2}-\frac{\sigma(\sigma-1)}{2} G(\phi)+\left(\sigma-\frac{\beta(\sigma+1)}{\alpha+2}\right)\|\phi\|_{L^{\alpha+2}}^{\alpha+2} \\
& \leq\left(\sigma+\frac{\beta(\beta-\sigma-2)}{\alpha+2}\right)\|\phi\|_{L^{\alpha+2}}^{\alpha+2} .
\end{aligned}
$$

In particular,

$$
\omega\|\phi\|_{L^{2}}^{2} \leq\left(1+\frac{\beta(\beta-\sigma-2)}{\sigma(\alpha+2)}\right)\|\phi\|_{L^{\alpha+2}}^{\alpha+2} .
$$

Since $K_{\omega}\left(v^{\lambda_{0}}\right)=0$, by Proposition 1.4, we have

$$
\frac{\alpha}{2(\alpha+2)}\|\phi\|_{L^{\alpha+2}}^{\alpha+2}=S_{\omega}(\phi) \leq S_{\omega}\left(v^{\lambda_{0}}\right)=\frac{\alpha}{2(\alpha+2)}\left\|v^{\lambda_{0}}\right\|_{L^{\alpha+2}}^{\alpha+2}=\frac{\alpha}{2(\alpha+2)} \lambda_{0}^{\beta}\|v\|_{L^{\alpha+2}}^{\alpha+2} .
$$

We thus get from (3.9) and the assumption $\|v\|_{L^{2}} \leq\|\phi\|_{L^{2}}$ that

$$
\omega\|v\|_{L^{2}}^{2} \leq\left(1+\frac{\beta(\beta-\sigma-2)}{\sigma(\alpha+2)}\right) \lambda_{0}^{\beta}\|v\|_{L^{\alpha+2}}^{\alpha+2} .
$$

We also have from $K_{\omega}\left(v^{\lambda_{0}}\right)=0,(3.10)$ and $Q(v) \leq 0$ that

$$
\begin{aligned}
G(v) & =\lambda_{0}^{2-\sigma}\|\nabla v\|_{L^{2}}^{2}+\lambda_{0}^{-\sigma} \omega\|v\|_{L^{2}}^{2}-\lambda_{0}^{\beta-\sigma}\|v\|_{L^{\alpha+2}}^{\alpha+2} \\
& \leq \lambda_{0}^{2-\sigma}\|\nabla v\|_{L^{2}}^{2}+\frac{\beta(\beta-\sigma-2)}{\sigma(\alpha+2)} \lambda_{0}^{\beta-\sigma}\|v\|_{L^{\alpha+2}}^{\alpha+2} \\
& \leq \frac{\sigma}{2} \lambda_{0}^{2-\sigma} G(v)+\frac{\beta}{\alpha+2}\left(\lambda_{0}^{2-\sigma}+\frac{\beta-\sigma-2}{\sigma} \lambda_{0}^{\beta-\sigma}\right)\|v\|_{L^{\alpha+2}}^{\alpha+2} .
\end{aligned}
$$

Thus,

$$
G(v) \leq \frac{2 \beta}{(\alpha+2)\left(2-\sigma \lambda_{0}^{2-\sigma}\right)}\left(\lambda_{0}^{2-\sigma}+\frac{\beta-\sigma-2}{\sigma} \lambda_{0}^{\beta-\sigma}\right)\|v\|_{L^{\alpha+2}}^{\alpha+2} .
$$

In view of (3.7) and (3.11), it suffices to show that

$$
\frac{\beta}{2-\sigma \lambda_{0}^{2-\sigma}}\left(\lambda_{0}^{2-\sigma}+\frac{\beta-\sigma-2}{\sigma} \lambda_{0}^{\beta-\sigma}\right) \leq \frac{2 \lambda_{0}^{\beta}-\beta \lambda_{0}^{2}-2+\beta}{\sigma \lambda_{0}^{2}-2 \lambda_{0}^{\sigma}-\sigma+2}
$$

which is equivalent to

$$
g\left(\lambda_{0}\right):=\frac{\left(2-\sigma \lambda_{0}^{2-\sigma}\right)\left(2 \lambda_{0}^{\beta}-\beta \lambda_{0}^{2}-2+\beta\right)}{\beta \lambda_{0}^{\beta-\sigma}\left(\sigma \lambda_{0}^{2}-2 \lambda_{0}^{\sigma}-\sigma+2\right)}-\frac{1}{\lambda_{0}^{\beta-2}}-\frac{\beta-\sigma-2}{\sigma} \geq 0 .
$$


Since $\lim _{\lambda \rightarrow 1} g(\lambda)=0$, the above inequality follows if we have

$$
\begin{aligned}
g^{\prime}(\lambda)=\frac{2\left(1-\lambda^{2-\sigma}\right)}{\beta \lambda^{\beta-\sigma+1}\left(\sigma \lambda^{2}-2 \lambda^{\sigma}-\sigma+2\right)^{2}} & {\left[2 \sigma(2-\sigma) \lambda^{\beta}-\sigma \beta(\beta-\sigma) \lambda^{2}\right.} \\
+ & \left.+2 \beta(\beta-2) \lambda^{\sigma}-(\beta-\sigma)(\beta-2)(2-\sigma)\right] \leq 0
\end{aligned}
$$

for all $\lambda \in(0,1)$. The above inequality holds if we have

$$
g_{1}(\lambda):=2 \sigma(2-\sigma) \lambda^{\beta}-\sigma \beta(\beta-\sigma) \lambda^{2}+2 \beta(\beta-2) \lambda^{\sigma}-(\beta-\sigma)(\beta-2)(2-\sigma) \leq 0
$$

for all $\lambda \in(0,1)$. Since $g_{1}(1)=0$, it is enough to show that

$$
g_{1}^{\prime}(\lambda)=2 \sigma \beta \lambda^{\sigma-1}\left[(2-\sigma) \lambda^{\beta-\sigma}-(\beta-\sigma) \lambda^{2-\sigma}+\beta-2\right] \geq 0
$$

for all $\lambda \in(0,1)$. This is equivalent to

$$
g_{2}(\lambda):=(2-\sigma) \lambda^{\beta-\sigma}-(\beta-\sigma) \lambda^{2-\sigma}+\beta-2 \geq 0
$$

for all $\lambda \in(0,1)$. Since $g_{2}(1)=0$ and

$$
g_{2}^{\prime}(\lambda)=-(\beta-\sigma)(2-\sigma) \lambda^{1-\sigma}\left(1-\lambda^{\beta-2}\right) \leq 0,
$$

we have $g_{2}(\lambda) \geq 0$ for all $\lambda \in(0,1)$. Therefore, we obtain $f\left(\lambda_{0}\right) \leq f(1)$ and the proof is complete.

Lemma 3.4. Let $d \geq 1,0<\sigma<\min \{2, d\}, \frac{4}{d}<\alpha<\frac{4}{d-2}$ if $d \geq 3\left(\frac{4}{d}<\alpha<\infty\right.$ if $\left.d=1,2\right)$ and $\omega>-\mu_{1}$. Let $\phi \in \mathcal{G}_{\omega}$ be such that $\left.\partial_{\lambda}^{2} S_{\omega}\left(\phi^{\lambda}\right)\right|_{\lambda=1} \leq 0$, where $\phi^{\lambda}$ is as in (3.1). Then the sets $\mathcal{K}_{\omega}^{ \pm}$are invariant under the flow of the focusing problem (1.1).

Proof. We only consider the case $\mathcal{K}_{\omega}^{-}$, the one for $\mathcal{K}_{\omega}^{+}$is similar. Let $u_{0} \in \mathcal{K}_{\omega}^{-}$, i.e. $\left\|u_{0}\right\|_{L^{2}} \leq\|\phi\|_{L^{2}}$, $S_{\omega}\left(u_{0}\right)<S_{\omega}(\phi), K_{\omega}\left(u_{0}\right)<0$ and $Q\left(u_{0}\right)<0$. We will show that $u(t) \in \mathcal{K}_{\omega}^{-}$for any $t$ in the existence time. By the conservation of mass and energy, we have

$$
\|u(t)\|_{L^{2}}=\left\|u_{0}\right\|_{L^{2}} \leq\|\phi\|_{L^{2}}, \quad S_{\omega}(u(t))=S_{\omega}\left(u_{0}\right)<S_{\omega}(\phi)
$$

for any $t \in I_{\max }$, where $I_{\max }$ is the maximal existence time interval. Let us prove $K_{\omega}(u(t))<0$ for any $t \in I_{\max }$. Suppose that there exists $t_{0} \in I_{\max }$ such that $K_{\omega}\left(u\left(t_{0}\right)\right) \geq 0$. By the continuity of $t \mapsto K_{\omega}(u(t))$, there exists $t_{1} \in I_{\max }$ such that $K_{\omega}\left(u\left(t_{1}\right)\right)=0$. By Proposition 1.4, $S_{\omega}\left(u\left(t_{1}\right)\right) \geq S_{\omega}(\phi)$ which contradicts to (3.12). We finally prove $Q(u(t))<0$ for any $t \in I_{\max }$. Suppose it is not true, then there exists $t_{2} \in I_{\max }$ such that $Q\left(u\left(t_{2}\right)\right) \geq 0$. By continuity of $t \mapsto Q(u(t))$, there exists $t_{3} \in I_{\max }$ such that $Q\left(u\left(t_{3}\right)\right)=0$. We thus obtain a function $u\left(t_{3}\right) \in H^{1} \backslash\{0\}$ satisfying $\left\|u\left(t_{3}\right)\right\|_{L^{2}} \leq\|\phi\|_{L^{2}}, S_{\omega}\left(u\left(t_{3}\right)\right)<S_{\omega}(\phi), K_{\omega}\left(u\left(t_{3}\right)\right)<0$ and $Q\left(u\left(t_{3}\right)\right)=0$. This is not possible due to (3.6). The proof is complete.

Proposition 3.5. Let $d \geq 1,0<\sigma<\min \{2, d\}, \frac{4}{d}<\alpha<\frac{4}{d-2}$ if $d \geq 3\left(\frac{4}{d}<\alpha<\infty\right.$ if $d=1$,2) and $\omega>-\mu_{1}$. Let $\phi \in \mathcal{G}_{\omega}$ be such that $\left.\partial_{\lambda}^{2} S_{\omega}\left(\phi^{\lambda}\right)\right|_{\lambda=1} \leq 0$, where $\phi^{\lambda}$ is as in (3.1).

- If $u_{0} \in \mathcal{K}_{\omega}^{-}$and $|x| u_{0} \in L^{2}$, then the corresponding solution to (1.1) blows up in finite time.

- If $u_{0} \in \mathcal{K}_{\omega}^{+}$, then the corresponding solution to (1.1) exists globally in time.

Proof. Let us first consider the case $u_{0} \in \mathcal{K}_{\omega}^{-}$and $|x| u_{0} \in L^{2}$. It is well-known that $|x| u(t) \in L^{2}$ for all $t \in I_{\max }$. By (1.9) and the convexity argument of Glassey [16], it suffices to show there exists $\delta>0$ such that

$$
Q(u(t)) \leq-\delta, \quad \forall t \in I_{\max } .
$$

To do so, we note that since $\mathcal{K}_{\omega}^{-}$is invariant under the flow of (1.1), $u(t) \in \mathcal{K}_{\omega}^{-}$for all $t \in I_{\max }$, i.e. $\|u(t)\|_{L^{2}} \leq\|\phi\|_{L^{2}}, S_{\omega}(u(t))<S_{\omega}(\phi), K_{\omega}(u(t))<0$ and $Q(u(t))<0$ for all $t \in I_{\max }$. Applying Proposition 3.2 to $u(t)$, we get

$$
Q(u(t)) \leq 2\left(S_{\omega}(u(t))-S_{\omega}(\phi)\right)=2\left(S_{\omega}\left(u_{0}\right)-S_{\omega}(\phi)\right),
$$

for all $t \in I_{\max }$, where we have used the conservation of mass and energy. This shows (3.13) with $\delta:=$ $2\left(S_{\omega}(\phi)-S_{\omega}\left(u_{0}\right)\right)>0$.

We now consider $u_{0} \in \mathcal{K}_{\omega}^{+}$. By the local well-posedness, it suffices to show there exists $C>0$ such that

$$
\|u(t)\|_{H^{1}} \leq C, \quad \forall t \in I_{\max }
$$


By Lemma 3.4, $u(t) \in \mathcal{K}_{\omega}^{+}$for all $t \in I_{\max }$, i.e. $\|u(t)\|_{L^{2}} \leq\|\phi\|_{L^{2}}, S_{\omega}(u(t))<S_{\omega}(\phi), K_{\omega}(u(t))<0$ and $Q(u(t))>0$ for all $t \in I_{\max }$. We have

$$
\frac{1}{2} H_{\omega}(u(t))-\frac{1}{\beta} Q(u(t))=\left(\frac{1}{2}-\frac{1}{\beta}\right)\|\nabla u(t)\|_{L^{2}}^{2}+\frac{\omega}{2}\|u(t)\|_{L^{2}}^{2}-\frac{\beta-\sigma}{2 \beta} G(u(t))+\frac{1}{\alpha+2}\|u(t)\|_{L^{\alpha+2}}^{\alpha+2} .
$$

It follows that

$$
\begin{aligned}
\left(\frac{1}{2}-\frac{1}{\beta}\right)\|\nabla u(t)\|_{L^{2}}^{2} & =\frac{1}{2} H_{\omega}(u(t))-\frac{1}{\alpha+2}\|u(t)\|_{L^{\alpha+2}}^{\alpha+2}-\frac{1}{\beta} Q(u(t))-\frac{\omega}{2}\|u(t)\|_{L^{2}}^{2}+\frac{\beta-\sigma}{2 \beta} G(u(t)) \\
& =S_{\omega}(u(t))-\frac{1}{\beta} Q(u(t))-\frac{\omega}{2}\|u(t)\|_{L^{2}}^{2}+\frac{\beta-\sigma}{2 \beta} G(u(t)) \\
& <S_{\omega}(\phi)-\frac{\omega}{2}\|u(t)\|_{L^{2}}^{2}+\frac{\beta-\sigma}{2 \beta} G(u(t)) .
\end{aligned}
$$

By Hardy's inequality (2.3) and the fact $0<\sigma<2$, we apply the Young's inequality to have for any $\varepsilon>0$,

$$
G(u(t)) \leq \varepsilon\|\nabla u(t)\|_{L^{2}}^{2}+C(\varepsilon)\|u(t)\|_{L^{2}}^{2} .
$$

We thus have that

$$
\left(\frac{1}{2}-\frac{1}{\beta}-\varepsilon\right)\|\nabla u(t)\|_{L^{2}}^{2}<S_{\omega}(\phi)+\left(\frac{(\beta-\sigma) C(\varepsilon)}{2 \beta}-\frac{\omega}{2}\right)\|u(t)\|_{L^{2}}^{2}
$$

for all $t \in I_{\max }$. Since we are considering the $L^{2}$-supercritical case, we see that $\beta>2$. By choosing $0<\varepsilon<\frac{1}{2}-\frac{1}{\beta}$ and using the conservation of mass, we prove (3.14). The proof is complete.

Remark 3.6. It is expected that the same finite time blow-up holds for radially symmetric initial data in $\mathcal{K}_{\omega}^{-}$. However, in the presence of inverse-power potentials with $0<\sigma<2$, it is not clear how to show it at the moment. In fact, by radial Sobolev embeddings (see e.g. [13]), it suffices to show that for $\varepsilon>0$ small enough, there exists $\delta(\varepsilon)>0$ such that

$$
Q(u(t))+\varepsilon\|\nabla u(t)\|_{L^{2}}^{2} \leq-\delta(\varepsilon)
$$

for any $t \in I_{\max }$. Note that

$$
S_{\omega}(v)=\frac{1}{\beta} Q(v)+\frac{\beta-2}{2 \beta}\|\nabla v\|_{L^{2}}^{2}+\frac{\omega}{2}\|v\|_{L^{2}}^{2}-\frac{\beta-2}{2 \beta} G(v)=: \frac{1}{\beta} Q(v)+P_{\omega}(v)
$$

and

It follows that

$$
\|\nabla v\|_{L^{2}}^{2}=\frac{2 \beta}{\beta-2} P_{\omega}(v)-\frac{\omega \beta}{\beta-2}\|v\|_{L^{2}}^{2}+\frac{\beta-\sigma}{\beta-2} G(v) .
$$

$$
\begin{aligned}
Q(v)+\varepsilon\|\nabla v\|_{L^{2}}^{2} & =\beta S_{\omega}(v)-\beta P_{\omega}(v)+\varepsilon\|\nabla v\|_{L^{2}}^{2} \\
& =\beta S_{\omega}(v)-\beta\left(1-\frac{2 \varepsilon}{\beta-2}\right) P_{\omega}(v)-\frac{\omega \beta \varepsilon}{\beta-2}\|v\|_{L^{2}}^{2}+\frac{(\beta-\sigma) \varepsilon}{\beta-2} G(v) \\
& \leq \beta(1-\rho) S_{\omega}(\phi)-\beta\left(1-\frac{2 \varepsilon}{\beta-2}\right) P_{\omega}(v)+\frac{(\beta-\sigma) \varepsilon}{\beta-2} G(v),
\end{aligned}
$$

where we have use $S_{\omega}(v) \leq(1-\rho) S_{\omega}(\phi)$ for some $\rho>0$ due to $S_{\omega}(v)<S_{\omega}(\phi)$. In the case of no potential, i.e. $c=0$ or $G(v)=0$, we can show that

$$
S_{\omega}(\phi)=\inf \left\{P_{\omega}(v): v \in H^{1} \backslash\{0\}, Q(v)=0\right\} .
$$

Note that since $Q(v)<0$, there exists $\lambda_{0} \in(0,1)$ such that $Q\left(\lambda_{0} v\right)=0$. Thus

$$
S_{\omega}(\phi) \leq P_{\omega}\left(\lambda_{0} v\right)=\lambda_{0}^{2} P_{\omega}(v)<P_{\omega}(v) .
$$

This shows that

$$
Q(v)+\varepsilon\|\nabla v\|_{L^{2}}^{2} \leq-\beta\left(\rho-\frac{2 \varepsilon}{\beta-2}\right) S_{\omega}(\phi),
$$

and (3.16) holds with $\delta(\varepsilon)=\beta\left(\rho-\frac{2 \varepsilon}{\beta-2}\right) S_{\omega}(\phi)>0$. In the case of inverse-power potentials with $0<\sigma<2$, we do not have (3.17), and there is an additional positive term $\frac{(\beta-\sigma) \varepsilon}{\beta-2} G(v)$ which is difficult to control. 
Lemma 3.7 ( [15]). There exists $\omega_{0}>-\mu_{1}$ such that if $\omega \geq \omega_{0}$ and $\phi_{\omega} \in \mathcal{G}_{\omega}$, then $\left.\partial_{\lambda}^{2} S_{\omega}\left(\phi_{\omega}^{\lambda}\right)\right|_{\lambda=1} \leq 0$, where $\phi_{\omega}^{\lambda}$ is as in (3.1).

We refer the readers to [15, Section 2] for the proof of this result.

Proof of Theorem 1.5. It follows directly from Proposition 3.5 and Lemma 3.7.

\section{Existence AND StABILITy of STANDing WAVES}

In this section, we give the proof of Theorem 1.8 and 1.9 .

Proof of Theorem 1.8. The proof is divided in several steps.

Step 1. We will show that the minimizing problem (1.12) is well-defined and there exists $C>0$ such that $I(a) \leq-C<0$. Indeed, let $v \in H^{1}$ be such that $\|v\|_{L^{2}}^{2}=a$. By Hardy's inequality and Young's inequality with $0<\sigma<2$ (see (3.15)), we have for any $\varepsilon>0$,

$$
G(v) \leq \varepsilon\|\nabla v\|_{L^{2}}^{2}+C(\varepsilon)\|v\|_{L^{2}}^{2}=\varepsilon\|\nabla v\|_{L^{2}}^{2}+C(\varepsilon) a .
$$

By the Gagliardo-Nirenberg inequality,

$$
\|v\|_{L^{\alpha+2}}^{\alpha+2} \lesssim\|\nabla v\|_{L^{2}}^{\frac{d \alpha}{2}}\|v\|_{L^{2}}^{\frac{4-(d-2) \alpha}{L^{2}}} .
$$

We next apply the Young's inequality with the fact $0<\frac{d \alpha}{2}<2$ to get for any $\varepsilon>0$,

$$
\frac{1}{\alpha+2}\|v\|_{L^{\alpha+2}}^{\alpha+2} \leq \frac{\varepsilon}{2}\|\nabla v\|_{L^{2}}^{2}+C(\varepsilon, \alpha, a)
$$

This shows that for any $\varepsilon>0$, there exists $C(\varepsilon, \alpha, a)>0$ such that

$$
E(v) \geq\left(\frac{1}{2}-\varepsilon\right)\|\nabla v\|_{L^{2}}^{2}-C(\varepsilon, \alpha, a) .
$$

By choosing $0<\varepsilon<\frac{1}{2}$, we see that $E(v) \geq-C(\varepsilon, \alpha, a)$. Thus the minimizing problem (1.12) is well-defined. Let $v^{\lambda}$ be as in (3.1). It is easy to check that $\left\|v^{\lambda}\right\|_{L^{2}}^{2}=\|v\|_{L^{2}}^{2}=a$ and

$$
E\left(v^{\lambda}\right)=\frac{\lambda^{2}}{2}\|\nabla v\|_{L^{2}}^{2}-\frac{\lambda^{\sigma}}{2} G(v)-\frac{\lambda^{\beta}}{\alpha+2}\|v\|_{L^{\alpha+2}}^{\alpha+2},
$$

where $\beta$ is as in (1.8). Since $0<\sigma<2$ and $0<\beta<2$, we can find $\lambda_{0}>0$ small enough so that $E\left(v^{\lambda_{0}}\right)<0$. Taking $C=-E\left(v^{\lambda_{0}}\right)>0$, we obtain that $I(a) \leq-C<0$.

Step 2. We will show that $\mathcal{N}(a) \neq \emptyset$. Let $\left(v_{n}\right)_{n \geq 1}$ be a minimizing sequence for $I(a)$, i.e. $\left\|v_{n}\right\|_{L^{2}}^{2}=a$ for all $n \geq 1$ and $E\left(v_{n}\right) \rightarrow I(a)$ as $n \rightarrow \infty$. By the same argument as in the proof of Lemma 2.3, we may assume that $\left(v_{n}\right)_{n \geq 1}$ is a radially symmetric and radially decreasing sequence. Since $E\left(v_{n}\right) \rightarrow I(a)$ as $n \rightarrow \infty$, there exists $C>0$ such that $E\left(v_{n}\right) \leq I(a)+C$ for any $n \geq 1$. By (4.2),

$$
\left(\frac{1}{2}-\varepsilon\right)\left\|\nabla v_{n}\right\|_{L^{2}}^{2} \leq E\left(v_{n}\right)+C(\varepsilon, \alpha, a) \leq I(a)+C(\varepsilon, \alpha, a) .
$$

Taking $0<\varepsilon<\frac{1}{2}$, we infer that $\left(v_{n}\right)_{n \geq 1}$ is a bounded sequence in $H_{\mathrm{rd}}^{1}$. Thanks to (1.16), there exist $v \in H^{1}$ and a subsequence still denoted by $\left(v_{n}\right)_{n \geq 1}$ such that $v_{n} \rightarrow v$ weakly in $H^{1}$ and $v_{n} \rightarrow v$ strongly in $L^{q}$ for any $2<q<\frac{2 d}{d-2}$ if $d \geq 3(2<q<\infty$ if $d=1,2)$.

Since $G\left(v_{n}\right) \rightarrow G(v)$ as $n \rightarrow \infty$ (see again the proof of Lemma 2.3), we see that $v \neq 0$. In fact, assume by contradiction that $v \equiv 0$. Since $v_{n} \rightarrow 0$ weakly in $H^{1}, v_{n} \rightarrow 0$ strongly in $L^{\alpha+2}$ and $G\left(v_{n}\right) \rightarrow 0$ as $n \rightarrow \infty$, we learn from Step 1 that

$$
0 \leq \liminf _{n \rightarrow \infty} \frac{1}{2}\left\|\nabla v_{n}\right\|_{L^{2}}^{2}=\liminf _{n \rightarrow \infty} E\left(v_{n}\right)+\frac{G\left(v_{n}\right)}{2}+\frac{1}{\alpha+2}\left\|v_{n}\right\|_{L^{\alpha+2}}^{\alpha+2}=I(a) \leq-C<0
$$

which is a contradiction. We also have that

$$
E(v) \leq \liminf _{n \rightarrow \infty} E\left(v_{n}\right)=I(a) .
$$

We next show that the minimizing problem (1.12) is attained by $v$. To see this, we write

$$
v_{n}(x)=v(x)+r_{n}(x)
$$


where $r_{n} \rightarrow 0$ weakly in $H^{1}$ and $r_{n} \rightarrow 0$ strongly in $L^{q}$ with $2<q<\frac{2 d}{d-2}$ if $d \geq 3(2<q<\infty$ if $d=1,2)$. We have the following expansions:

$$
\begin{aligned}
\left\|v_{n}\right\|_{L^{2}}^{2} & =\|v\|_{L^{2}}^{2}+\left\|r_{n}\right\|_{L^{2}}^{2}+o_{n}(1) \\
\left\|\nabla v_{n}\right\|_{L^{2}}^{2} & =\|\nabla v\|_{L^{2}}^{2}+\left\|\nabla r_{n}\right\|_{L^{2}}^{2}+o_{n}(1) \\
\left\|v_{n}\right\|_{L^{\alpha+2}}^{\alpha+2} & =\|v\|_{L^{\alpha+2}}^{\alpha+2}+\left\|r_{n}\right\|_{L^{\alpha+2}}^{\alpha+2}+o_{n}(1) \\
G\left(v_{n}\right) & =G(v)+G\left(r_{n}\right)+o_{n}(1)
\end{aligned}
$$

as $n \rightarrow \infty$. In particular, we have

$$
E\left(v_{n}\right)=E(v)+E\left(r_{n}\right)+o_{n}(1)
$$

as $n \rightarrow \infty$. The expansions (4.4), (4.5) and (4.6) are standard. We thus only prove (4.7). To see this, we write

We will show that

$$
G\left(v_{n}\right)=G(v)+G\left(r_{n}\right)+2 c \int|x|^{-\sigma} \operatorname{Re}\left(v(x) \bar{r}_{n}(x)\right) d x .
$$

$$
\int|x|^{-\sigma} v(x) \bar{r}_{n}(x) d x \rightarrow 0
$$

as $n \rightarrow \infty$. Without loss of generality, we may assume that $v$ is continuous and compactly supported.

In the case $0 \notin \operatorname{supp}(v)$, we have

$$
\left.\left|\int\right| x\right|^{-\sigma} v(x) \bar{r}_{n}(x) d x\left|\leq\left\||\cdot|^{-\sigma} v\right\|_{L^{\infty}} \int_{\operatorname{supp}(v)}\right| r_{n}(x) \mid d x \rightarrow 0
$$

as $n \rightarrow \infty$. Here we have used the fact $r_{n} \rightarrow 0$ weakly in $H^{1}$ and the compact embedding $H^{1} \hookrightarrow L_{\text {loc }}^{1}$ to show $r_{n} \rightarrow 0$ strongly in $L_{\text {loc }}^{1}$.

In the case $0 \in \operatorname{supp}(v)$, let $\varepsilon>0$. For $\eta>0$ small to be chosen later, we estimate

$$
\left.\left.\left|\int\right| x\right|^{-\sigma} v(x) \bar{r}_{n}(x) d x\left|\leq\left[\int_{B(0, \eta)}+\int_{\operatorname{supp}(v) \backslash B(0, \eta)}\right]\right| x\right|^{-\sigma}|v(x)|\left|r_{n}(x)\right| d x=: J_{1}+J_{2} .
$$

The term $J_{2}$ is treated as above, and there exists $n_{0} \in \mathbb{N}$ such that for $n \geq n_{0}, J_{2}<\frac{\varepsilon}{2}$. For $J_{1}$, we use the Cauchy-Schwarz inequality and Hardy's inequality to have

$$
\begin{aligned}
J_{1} & \leq\left(\int_{B(0, \eta)}|x|^{-\sigma}|v(x)|^{2} d x\right)^{\frac{1}{2}}\left(\int|x|^{-\sigma}\left|r_{n}(x)\right|^{2} d x\right)^{\frac{1}{2}} \\
& \lesssim\left(\int_{B(0, \eta)}|x|^{-\sigma}|v(x)|^{2} d x\right)^{\frac{1}{2}}\left\|r_{n}\right\|_{H^{1}} .
\end{aligned}
$$

Since $\left(r_{n}\right)_{n \geq 1}$ is bounded in $H^{1}$ and $v \in H^{1}$, the dominated convergence implies that for $\eta>0$ small enough, $J_{1} \leq \frac{\varepsilon}{2}$. It follows that for $n \geq n_{0}$,

$$
\left.\left|\int\right| x\right|^{-\sigma} v(x) \bar{r}_{n}(x) d x \mid<\varepsilon
$$

Collecting the above two cases, we prove (4.9).

We now set $\tilde{v}=\lambda v$ and $\tilde{r}_{n}=\lambda_{n} r_{n}$, where

$$
\lambda:=\frac{\sqrt{a}}{\|v\|_{L^{2}}} \geq 1, \quad \lambda_{n}:=\frac{\sqrt{a}}{\left\|r_{n}\right\|_{L^{2}}} \geq 1 .
$$

It is obvious that $\|\tilde{v}\|_{L^{2}}^{2}=\left\|\tilde{r}_{n}\right\|_{L^{2}}^{2}=a$, hence $E(\tilde{v}) \geq I(a)$ and $E\left(\tilde{r}_{n}\right) \geq I(a)$. We also have that

$$
E(\tilde{v})=\frac{\lambda^{2}}{2}\|\nabla v\|_{L^{2}}^{2}-\frac{\lambda^{2}}{2} G(v)-\frac{\lambda^{\alpha+2}}{\alpha+2}\|v\|_{L^{\alpha+2}}^{\alpha+2} .
$$

This implies that

$$
E(v)=\frac{E(\tilde{v})}{\lambda^{2}}+\frac{\lambda^{\alpha}-1}{\alpha+2}\|v\|_{L^{\alpha+2}}^{\alpha+2},
$$


and

Using (4.8), we get

$$
E\left(r_{n}\right)=\frac{E\left(\tilde{r}_{n}\right)}{\lambda_{n}^{2}}+\frac{\lambda_{n}^{\alpha}-1}{\alpha+2}\left\|r_{n}\right\|_{L^{\alpha+2}}^{\alpha+2} \geq \frac{E\left(\tilde{r}_{n}\right)}{\lambda_{n}^{2}}
$$

$$
\begin{aligned}
E\left(v_{n}\right) & \geq \frac{E(\tilde{v})}{\lambda^{2}}+\frac{\lambda^{\alpha}-1}{\alpha+2}\|v\|_{L^{\alpha+2}}^{\alpha+2}+\frac{E\left(\tilde{r}_{n}\right)}{\lambda_{n}^{2}}+o_{n}(1) \\
& \geq \frac{\|v\|_{L^{2}}^{2}}{a} I(a)+\frac{\lambda^{\alpha}-1}{\alpha+2}\|v\|_{L^{\alpha+2}}^{\alpha+2}+\frac{\left\|r_{n}\right\|_{L^{2}}^{2}}{a} I(a)+o_{n}(1) \\
& =\frac{I(a)}{a}\left(\|v\|_{L^{2}}^{2}+\left\|r_{n}\right\|_{L^{2}}^{2}\right)+\frac{\lambda^{\alpha}-1}{\alpha+2}\|v\|_{L^{\alpha+2}}^{\alpha+2}+o_{n}(1) .
\end{aligned}
$$

Taking $n \rightarrow \infty$ and using (4.4) and the fact $v \neq 0$, we have that

$$
I(a) \geq I(a)+\frac{\lambda^{\alpha}-1}{\alpha+2}\|v\|_{L^{\alpha+2}}^{\alpha+2} .
$$

We thus obtain $\lambda \leq 1$, hence $\lambda=1$ hence $\|v\|_{L^{2}}^{2}=\left\|v_{n}\right\|_{L^{2}}^{2}=a$. This implies that

$$
E(v) \geq I(a) \text {. }
$$

By (4.3) and (4.10), we obtain $E(v)=I(a)$ and $\|v\|^{2}=a$ which implies that the minimizing problem (1.12) is attained by $v$ or $\mathcal{N}(a) \neq \emptyset$.

Moreover, we also have that $v_{n} \rightarrow v$ strongly in $H^{1}$. In fact, by (4.4) and $\|v\|_{L^{2}}^{2}=\left\|v_{n}\right\|_{L^{2}}^{2}=a$, we get $\left\|r_{n}\right\|_{L^{2}} \rightarrow 0$ as $n \rightarrow \infty$. Since $r_{n} \rightarrow 0$ weakly in $H^{1}$, by the uniqueness of the weak limit, $r_{n} \rightarrow 0$ strongly in $L^{2}$. By (4.8), $\lim _{n \rightarrow \infty} E\left(v_{n}\right)=I(a), E(v)=I(a)$, the fact $G\left(r_{n}\right) \rightarrow 0$ and $v_{n} \rightarrow 0$ strongly in $L^{\alpha+2}$, we see that $\lim _{n \rightarrow \infty}\left\|\nabla r_{n}\right\|_{L^{2}}^{2}=0$. This implies that $\lim _{n \rightarrow \infty}\left\|\nabla v_{n}\right\|=\|\nabla v\|_{L^{2}}$ which together with $v_{n} \rightarrow v$ weakly in $H^{1}$ imply $v_{n} \rightarrow v$ strongly in $\dot{H}^{1}$. Therefore, $v_{n} \rightarrow v$ strongly in $H^{1}$.

Step 3. Let $v \in H^{1}$ be a complex valued minimizer for $I(a)$. A standard elliptic regularity bootstrap ensures that $v$ is of class $C^{1}$. By the diamagnetic inequality, we see that $|v|$ is also a minimizer for $I(a)$. Moreover, by the Euler-Lagrange equation and using the strong maximum principle, we get $|v|>0$ and thus $v \in C^{1}\left(\mathbb{R}^{d}, \mathbb{C} \backslash\{0\}\right)$. Since $E(v)=E(|v|)=I(a)$, it follows that $\|\nabla(|v|)\|_{L^{2}}^{2}=\|\nabla v\|_{L^{2}}^{2}$. Set $w(x):=\frac{v(x)}{|v(x)|}$. It follows from the fact $|w(x)|^{2}=1$ for all $x \in \mathbb{R}^{d}$ that $\operatorname{Re}(\bar{w}(x) \nabla w(x))=0$,

$$
\nabla v(x)=\nabla(|v|)(x) w(x)+|v(x)| \nabla w(x),
$$

and thus $|\nabla v(x)|^{2}=|\nabla(|v|)(x)|^{2}+|v(x)|^{2}|\nabla w(x)|^{2}$ for all $x \in \mathbb{R}^{d}$. We also have from this and $\|\nabla(|v|)\|_{L^{2}}^{2}=$ $\|\nabla v\|_{L^{2}}^{2}$ that

$$
\int|v(x)|^{2}|\nabla w(x)|^{2} d x=0
$$

which implies $|\nabla w(x)|=0$ for all $x \in \mathbb{R}^{d}$. Hence $w$ is a constant with $|w|=1$. We infer that there exists $\theta \in \mathbb{R}$ such that $v(x)=e^{i \theta} \phi(x)$, where $\phi(x)=|v(x)|$. We next prove that $\phi$ is radially symmetric and radially decreasing. Let $\phi^{*}$ be the symmetric rearrangement of $\phi$. It is well-known (see e.g. [21]) that

$$
\int|x|^{-\sigma}\left|\phi^{*}(x)\right|^{2} d x>\int|x|^{-\sigma}|\phi(x)|^{2} d x \text { unless } \phi=\phi^{*} .
$$

By the Polya-Szego's inequality $\left\|\nabla \phi^{*}\right\|_{L^{2}} \leq\|\nabla \phi\|_{L^{2}}$ and the fact $\left\|\phi^{*}\right\|_{L^{\alpha+2}}=\|\phi\|_{L^{\alpha+2}}$, it follows that if $\phi \neq \phi^{*}$, then $E\left(\phi^{*}\right)<E(\phi)$ and $\left\|\phi^{*}\right\|_{L^{2}}^{2}=\|\phi\|_{L^{2}}^{2}=a$ which contradicts $I(a)=E(v)=E(\phi)$. Therefore, $\phi$ is radially symmetric and radially decreasing.

Step 4. We will show that $\mathcal{N}(a)$ is orbitally stable under the flow of the focusing problem (1.1). To see this, we argue by contradiction. Note that the existence of global solutions is proved in Theorem 1.1. Suppose that there exist sequences $\left(u_{0, n}\right)_{n \geq 1} \subset H^{1},\left(t_{n}\right)_{n \geq 1} \subset \mathbb{R}$ and $\varepsilon_{0}>0$ such that for all $n \geq 1$,

$$
\inf _{v \in S_{a}}\left\|u_{0, n}-v\right\|_{H^{1}}<\frac{1}{n}
$$

and

$$
\inf _{v \in S_{a}}\left\|u_{n}\left(t_{n}\right)-v\right\|_{H^{1}} \geq \varepsilon_{0}
$$


where $u_{n}(t)$ is the solution to (1.1) with initial data $u_{0, n}$. By (4.11), we see that for each $n \geq 1$, there exists $v_{n} \in S_{a}$ such that

$$
\left\|u_{0, n}-v_{n}\right\|_{H^{1}}<\frac{2}{n}
$$

We thus have a sequence $\left(v_{n}\right)_{n \geq 1} \subset S_{a}$. By Step 2, there exists $v \in S_{a}$ such that

$$
\lim _{n \rightarrow \infty}\left\|v_{n}-v\right\|_{H^{1}}=0
$$

By (4.13) and (4.14), we have $u_{0, n} \rightarrow v$ in $H^{1}$ as $n \rightarrow \infty$. It follows that

$$
\lim _{n \rightarrow \infty}\left\|u_{0, n}\right\|_{L^{2}}^{2}=\|v\|_{L^{2}}^{2}=a, \quad \lim _{n \rightarrow \infty} E\left(u_{0, n}\right)=E(v)=I(a) .
$$

Thanks to the conservation of mass and energy,

$$
\lim _{n \rightarrow \infty}\left\|u_{n}\left(t_{n}\right)\right\|_{L^{2}}^{2}=a, \quad \lim _{n \rightarrow \infty} E\left(u_{n}\left(t_{n}\right)\right)=I(a) .
$$

By the same argument as in Step 2, we prove as well that there exists $\tilde{v} \in S_{a}$ such that up to a subsequence, $\left(u_{n}\left(t_{n}\right)\right)_{n \geq 1}$ converges strongly to $\tilde{v}$ in $H^{1}$ which contradicts with (4.12). The proof of Theorem 1.8 is now complete.

Proof of Theorem 1.9. The proof is similar to the one of Theorem 1.8 except (4.1) which becomes

$$
\frac{1}{\frac{4}{d}+2}\|v\|_{L^{\frac{4}{d}+2}}^{\frac{4}{d}+2} \leq \frac{1}{2}\left(\frac{\|v\|_{L^{2}}}{\|Q\|_{L^{2}}}\right)^{\frac{4}{d}}\|\nabla v\|_{L^{2}}^{2} .
$$

Thus (4.2) is replaced by

$$
E(v) \geq \frac{1}{2}\left(1-\left(\frac{\|v\|_{L^{2}}}{\|Q\|_{L^{2}}}\right)^{\frac{4}{d}}-\varepsilon\right)\|\nabla v\|_{L^{2}}^{2}-C(\varepsilon) a .
$$

Since $\|v\|_{L^{2}}<\|Q\|_{L^{2}}$, we choose $0<\varepsilon<1-\left(\frac{\|v\|_{L^{2}}}{\|Q\|_{L^{2}}}\right)^{\frac{4}{d}}$ to get the lower bound for $E(v)$. The rest of the proof follows the same lines as in the proof of Theorem 1.8. Note that the existence of global solutions is given in Theorem 1.1.

\section{BlOW-UP BEHAVIOR OF STANDing WAVES}

In this subsection, we will prove the non-existence of minimizers for $I(a)$ with $a \geq a^{*}$ in the mass-critical case as well as the blow-up behavior of minimizers for $I(a)$ as $a \nearrow a^{*}$.

Proof of Theorem 1.10. The proof is done by several steps.

Step 1. We first show that there is no minimizer for $I(a)$ with $a \geq a^{*}$. To do this, we pick $\varphi \in C_{0}^{\infty}\left(\mathbb{R}^{d}\right)$ satisfying $0 \leq \varphi \leq 1, \varphi=1$ on $|x| \leq 1$ and denote

$$
v_{\tau}(x):=A_{\tau} \tau^{\frac{d}{2}} \varphi(x) Q_{0}(\tau x), \quad \tau>0,
$$

where $Q_{0}=\frac{Q}{\|Q\|_{L^{2}}}$ and $A_{\tau}>0$ is such that $\left\|v_{\tau}\right\|_{L^{2}}^{2}=a$ for all $\tau>0$. It follows that

$$
a A_{\tau}^{-2}=\int \varphi^{2}\left(\tau^{-1} x\right) Q_{0}^{2}(x) d x=1+\int\left(1-\varphi^{2}\left(\tau^{-1} x\right)\right) Q_{0}^{2}(x) d x
$$

due to $\left\|Q_{0}\right\|_{L^{2}}^{2}=1$. Since $Q_{0},\left|\nabla Q_{0}\right|=O\left(e^{-\delta|x|}\right)$ for some $\delta>0$ as $|x| \rightarrow \infty$, we see that for $\tau$ sufficiently large and $N>0$,

$$
\left|\int\left(1-\varphi^{2}\left(\tau^{-1} x\right)\right) Q_{0}^{2}(x) d x\right| \lesssim \int_{|x| \geq \tau} e^{-2 \delta|x|} d x \lesssim \int_{|x| \geq \tau}|x|^{-d-N} d x \lesssim \tau^{-N} .
$$

This shows that

$$
a A_{\tau}^{-2}=1+O\left(\tau^{-\infty}\right)
$$

as $\tau \rightarrow \infty$. Here $A=O\left(\tau^{-\infty}\right)$ means that $|A| \leq C \tau^{-N}$ for any $N>0$. We next compute

$$
\begin{aligned}
\left\|\nabla v_{\tau}\right\|_{L^{2}}^{2}=A_{\tau}^{2}\left(\int\left|\nabla \varphi\left(\tau^{-1} x\right)\right|^{2} Q_{0}^{2}(x) d x\right. & +\tau^{2} \int \varphi^{2}\left(\tau^{-1} x\right)\left|\nabla Q_{0}(x)\right|^{2} d x \\
& \left.+2 \tau \int \operatorname{Re}\left(\varphi\left(\tau^{-1} x\right) Q_{0}(x) \nabla \varphi\left(\tau^{-1} x\right) \cdot \nabla Q_{0}(x)\right) d x\right) .
\end{aligned}
$$


Estimating as above and using the fact $A_{\tau}^{2}=a+O\left(\tau^{-\infty}\right)$ as $\tau \rightarrow \infty$, we get

$$
\left\|\nabla v_{\tau}\right\|_{L^{2}}^{2}=\tau^{2} a\left\|\nabla Q_{0}\right\|_{L^{2}}^{2}+O\left(\tau^{-\infty}\right)
$$

as $\tau \rightarrow \infty$. Similarly,

$$
\begin{aligned}
\int|x|^{-\sigma}\left|v_{\tau}(x)\right|^{2} d x & =\tau^{\sigma} A_{\tau}^{2} \int|x|^{-\sigma} \varphi^{2}\left(\tau^{-1} x\right) Q_{0}^{2}(x) d x \\
& =\tau^{\sigma} a \int|x|^{-\sigma} Q_{0}^{2}(x) d x+O\left(\tau^{-\infty}\right)
\end{aligned}
$$

and

$$
\begin{aligned}
\left\|v_{\tau}\right\|_{L^{\frac{4}{d}+2}}^{\frac{4}{d}+2} & =\tau^{2} A_{\tau}^{\frac{4}{d}+2} \int\left(\varphi\left(\tau^{-1} x\right) Q_{0}(x)\right)^{\frac{4}{d}+2} d x \\
& =\tau^{2} a^{\frac{2}{d}+1} \int\left(Q_{0}(x)\right)^{\frac{4}{d}+2} d x+O\left(\tau^{-\infty}\right)
\end{aligned}
$$

as $\tau \rightarrow \infty$. This implies that

$$
\begin{aligned}
\frac{I(a)}{a} \leq \frac{E\left(v_{\tau}\right)}{a} & =\tau^{2}\left(\frac{1}{2}\left\|\nabla Q_{0}\right\|_{L^{2}}^{2}-\frac{a^{\frac{2}{d}}}{\frac{4}{d}+2}\left\|Q_{0}\right\|_{L^{\frac{4}{d}+2}}^{\frac{4}{d}+2}\right)-\frac{\tau^{\sigma}}{2} G\left(Q_{0}\right)+O\left(\tau^{-\infty}\right) \\
& =\frac{\tau^{2} d}{4}\left(1-\left(\frac{a}{a^{*}}\right)^{\frac{2}{d}}\right)-\frac{\tau^{\sigma}}{2} G\left(Q_{0}\right)+O\left(\tau^{-\infty}\right) \\
& =\frac{\tau^{2} d}{4} \beta_{a}-\frac{\tau^{\sigma}}{2} G\left(Q_{0}\right)+O\left(\tau^{-\infty}\right)
\end{aligned}
$$

as $\tau \rightarrow \infty$. Here we have used the fact that

$$
1=\left\|Q_{0}\right\|_{L^{2}}^{2}=\frac{2}{d}\left\|\nabla Q_{0}\right\|_{L^{2}}^{2}=\frac{2}{d+2}\|Q\|_{L^{2}}^{\frac{4}{d}}\left\|Q_{0}\right\|_{L^{\frac{4}{d}+2}}^{\frac{4}{d}+2}
$$

which follows from the following Pohozaev's identities

$$
\|Q\|_{L^{2}}^{2}=\frac{2}{d}\|\nabla Q\|_{L^{2}}^{2}=\frac{2}{d+2}\|Q\|_{L^{\frac{4}{d}+2}}^{\frac{4}{d}+2} .
$$

We infer from (5.1) that for $a \geq a^{*}$,

$$
\frac{I(a)}{a} \leq \frac{E\left(v_{\tau}\right)}{a} \rightarrow-\infty \text { as } \tau \rightarrow \infty
$$

which shows the non-existence of minimizers for $I(a)$ with $a \geq a^{*}$.

Step 2. Let $v_{a}$ be a non-negative minimizer for $I(a)$ with $0<a<a^{*}$. We will show that $v_{a}$ blows up as $a \nearrow a^{*}$ in the sense of (1.17). Assume by contradiction that $\left(v_{a}\right)_{a} \nearrow a^{*}$ is bounded in $H^{1}$. We can assume that $v_{a}$ is radially symmetric and radially decreasing. By the same argument as in the proof of Theorem 1.8, we show that there exists a minimizer for $I\left(a^{*}\right)$ which is a contradiction.

Step 3. We now claim that there exist two positive constants $m<M$ independent of $a$ such that for $0<a<a^{*}$

$$
-M \beta_{a}^{-\frac{\sigma}{2-\sigma}} \leq \frac{I(a)}{a} \leq-m \beta_{a}^{-\frac{\sigma}{2-\sigma}},
$$

where $\beta_{a}$ is as in (1.18). To see this, we first show that $\frac{I(a)}{a}$ is a decreasing function in $a$. Indeed, let $0<a \leq b$. We will show that $\frac{I(b)}{b} \leq \frac{I(a)}{a}$. Let $v \in H^{1}$ be such that $\|v\|_{L^{2}}^{2}=a$ and set $\lambda=\sqrt{\frac{b}{a}} \geq 1$. We see that $\|\lambda v\|_{L^{2}}^{2}=b$ and

$$
\begin{aligned}
E(\lambda v) & =\frac{\lambda^{2}}{2}\|\nabla v\|_{L^{2}}^{2}-\frac{\lambda^{2}}{2} G(v)-\frac{\lambda^{\alpha+2}}{\alpha+2}\|v\|_{L^{\alpha+2}}^{\alpha+2} \\
& =\lambda^{2} E(v)+\frac{\lambda^{2}\left(1-\lambda^{\alpha}\right)}{\alpha+2}\|v\|_{L^{\alpha+2}}^{\alpha+2} \leq \lambda^{2} E(v) .
\end{aligned}
$$

We then have from the definition of $I(b)$ that

$$
I(b) \leq E(\lambda v) \leq \lambda^{2} E(v) .
$$


Taking the infimum over all $v \in H^{1}$ with $\|v\|_{L^{2}}^{2}=a$, we obtain $I(b) \leq \frac{b}{a} I(a)$ which shows that $\frac{I(a)}{a}$ is a decreasing function in $a$. Thus, we only need to show (5.2) for $a$ close to $a^{*}$.

We now have from Hardy's inequality and Young's inequality with $0<\sigma<2$ that for any $\varepsilon>0$,

$$
G(v) \leq C\|\nabla v\|_{L^{2}}^{\sigma}\|v\|_{L^{2}}^{2-\sigma} \leq \varepsilon\|\nabla v\|_{L^{2}}^{2}+C(\sigma) \varepsilon^{-\frac{\sigma}{2-\sigma}}\|v\|_{L^{2}}^{2} .
$$

Note that the constant $C$ may change from line to line. The above estimate together with the sharp GagliardoNirenberg inequality (4.15) imply that

$$
E(v) \geq \frac{1}{2}\left(1-\left(\frac{\|v\|_{L^{2}}}{\|Q\|_{L^{2}}}\right)^{\frac{4}{d}}-\varepsilon\right)\|\nabla v\|_{L^{2}}^{2}-\frac{C(\sigma)}{2} \varepsilon^{-\frac{\sigma}{2-\sigma}}\|v\|_{L^{2}}^{2} .
$$

Let $v \in H^{1}$ be such that $\|v\|_{L^{2}}^{2}=a$. It follows that

$$
\begin{aligned}
E(v) & \geq \frac{1}{2}\left(1-\left(\frac{a}{a^{*}}\right)^{\frac{2}{d}}-\varepsilon\right)\|\nabla v\|_{L^{2}}^{2}-\frac{C(\sigma)}{2} \varepsilon^{-\frac{\sigma}{2-\sigma}} a \\
& =\frac{1}{2}\left(\beta_{a}-\varepsilon\right)\|\nabla v\|_{L^{2}}^{2}-\frac{C(\sigma)}{2} \varepsilon^{-\frac{\sigma}{2-\sigma}} a,
\end{aligned}
$$

where $\beta_{a}$ is as in (1.18). We take $\varepsilon=\frac{1}{2} \beta_{a}$ and get

$$
E(v) \geq-2^{\frac{-2+2 \sigma}{2-\sigma}} C(\sigma) \beta_{a}^{-\frac{\sigma}{2-\sigma}} a .
$$

Taking the infimum over all $v \in H^{1}$ with $\|v\|_{L^{2}}^{2}=a$, we prove the lower bound in (5.2) with $M=2^{\frac{-2+2 \sigma}{2-\sigma}} C(\sigma)$. To see the upper bound in (5.2), we choose $\tau=\lambda \beta_{a}^{-\frac{1}{2-\sigma}}$ in (5.1) with $\lambda>0$. Note that $\tau \rightarrow \infty$ as $a \nearrow a^{*}$ since $\beta_{a} \rightarrow 0$ as $a \nearrow a^{*}$. With this choice, (5.1) becomes

$$
\frac{I(a)}{a} \leq\left(\frac{\lambda^{2} d}{4}-\frac{\lambda^{\sigma}}{2} G\left(Q_{0}\right)\right) \beta_{a}^{-\frac{\sigma}{2-\sigma}}+o_{a} \nearrow a^{*}(1)
$$

for any $\lambda>0$. Since $0<\sigma<2$, there exists $\lambda_{0}>0$ sufficiently small so that $-2 m:=\frac{\lambda_{0}^{2} d}{4}-\frac{\lambda_{0}^{\sigma}}{2} G\left(Q_{0}\right)<0$ and $m<M$. Taking $a$ sufficiently close to $a^{*}$, we prove the upper bound in (5.2). We also have from (5.4) that

$$
\limsup _{a \nearrow a^{*}} \beta_{a}^{\frac{\sigma}{2-\sigma}} \frac{I(a)}{a} \leq \inf _{\lambda>0}\left(\frac{\lambda^{2} d}{4}-\frac{\lambda^{\sigma}}{2} G\left(Q_{0}\right)\right) .
$$

Step 4. Let $v_{a}$ be a non-negative minimizer for $I(a)$ with $0<a<a^{*}$. We claim that then there exists $K>1$ independent of $a$ such that for $0<a<a^{*}$,

and

$$
-K \beta_{a}^{-\frac{\sigma}{2-\sigma}} \leq-\frac{G\left(v_{a}\right)}{a} \leq-\frac{1}{K} \beta_{a}^{-\frac{\sigma}{2-\sigma}}
$$

a

$$
\frac{\left\|\nabla v_{a}\right\|_{L^{2}}^{2}}{a} \leq K \beta_{a}^{-\frac{2}{2-\sigma}}
$$

The upper bound in (5.6) follows easily from the upper bound in (5.2) and the fact

$$
I(a)=E\left(v_{a}\right) \geq \frac{1}{2}\left(1-\left(\frac{a}{a^{*}}\right)^{\frac{2}{d}}\right)\left\|\nabla v_{a}\right\|_{L^{2}}^{2}-\frac{G\left(v_{a}\right)}{2} \geq-\frac{G\left(v_{a}\right)}{2} .
$$

To see the lower bound in (5.6), we use again (5.3) and the fact $E\left(v_{a}\right)=I(a)<0$ to have that

$$
\begin{aligned}
-\frac{G\left(v_{a}\right)}{2} \geq E\left(v_{a}\right)-\frac{G\left(v_{a}\right)}{2} & \geq \frac{1}{2}\left(1-\left(\frac{a}{a^{*}}\right)^{\frac{2}{d}}-2 \varepsilon\right)\left\|\nabla v_{a}\right\|_{L^{2}}^{2}-C(\sigma) \varepsilon^{-\frac{\sigma}{2-\sigma} a} \\
& =\frac{1}{2}\left(\beta_{a}-2 \varepsilon\right)\left\|\nabla v_{a}\right\|_{L^{2}}^{2}-C(\sigma) \varepsilon^{-\frac{\sigma}{2-\sigma}} a .
\end{aligned}
$$

We take $\varepsilon=\frac{1}{4} \beta_{a}$ and get

$$
-\frac{G\left(v_{a}\right)}{a} \geq \frac{1}{4} \beta_{a}\left\|\nabla v_{a}\right\|_{L^{2}}^{2}-2^{\frac{2+\sigma}{2-\sigma}} C(\sigma) \beta_{a}^{-\frac{\sigma}{2-\sigma}} \geq-2^{\frac{2+\sigma}{2-\sigma}} C(\sigma) \beta_{a}^{-\frac{\sigma}{2-\sigma}} .
$$

The above estimate also gives (5.7). 
Step 5. We finally show the blow-up behavior of minimizers for $I(a)$ as $a \nearrow a^{*}$. To this end, we denote

$$
w_{a}(x):=\beta_{a}^{\frac{d}{2(2-\sigma)}} v_{a}\left(\beta_{a}^{\frac{1}{2-\sigma}} x\right) .
$$

It follows that

and

$$
\left\|w_{a}\right\|_{L^{2}}^{2}=\left\|v_{a}\right\|_{L^{2}}^{2}=a
$$

$$
\left\|\nabla w_{a}\right\|_{L^{2}}^{2}=\beta_{a}^{\frac{2}{2-\sigma}}\left\|\nabla v_{a}\right\|_{L^{2}}^{2} \leq K a
$$

by (5.7). This implies that $\left(w_{a}\right)_{a \nearrow a^{*}}$ is a bounded sequence in $H^{1}$. Up to a subsequence, $w_{a} \rightarrow w$ weakly in $H^{1}$ and pointwise almost everywhere. By the lower continuity of the weak limit,

$$
\|w\|_{L^{2}}^{2} \leq \liminf _{a \nearrow a^{*}}\left\|w_{a}\right\|_{L^{2}}^{2}=a^{*} .
$$

By (5.6) and the weak continuity of the potential energy (see e.g. [26, Theorem 11.4]),

as $a \nearrow a^{*}$. This shows that $w \neq 0$.

$$
\frac{1}{K} \leq \frac{\beta_{a}^{\frac{\sigma}{2-\sigma}} G\left(v_{a}\right)}{a}=\frac{G\left(w_{a}\right)}{a} \rightarrow \frac{G(w)}{a^{*}}
$$

We next show that $w$ is actually a non-negative optimizer for the sharp Gagliardo-Nirenberg inequality (1.19). In fact, we have from (5.2), (5.6) and the fact $0<\sigma<2$ that

$$
\begin{aligned}
0 & =\lim _{a \nearrow a^{*}} \beta_{a}^{\frac{2}{2-\sigma}}\left(\frac{E\left(v_{a}\right)}{a}+\frac{G\left(v_{a}\right)}{2 a}\right) \\
& =\lim _{a \nearrow a^{*}} \frac{\beta_{a}^{\frac{2}{2-\sigma}}}{a}\left(\frac{1}{2}\left\|\nabla v_{a}\right\|_{L^{2}}^{2}-\frac{1}{\frac{4}{d}+2}\left\|v_{a}\right\|_{L^{\frac{4}{d}+2}}^{\frac{4}{d}+2}\right) \\
& =\lim _{a \nearrow a^{*}} \frac{1}{a}\left(\frac{1}{2}\left\|\nabla w_{a}\right\|_{L^{2}}^{2}-\frac{1}{\frac{4}{d}+2}\left\|w_{a}\right\|_{L^{\frac{4}{d}+2}}^{\frac{4}{d}+2}\right) .
\end{aligned}
$$

Since $w_{a} \rightarrow w$ weakly in $H^{1}$, we have

$$
\begin{aligned}
\left\|w_{a}\right\|_{L^{2}}^{2} & =\|w\|_{L^{2}}^{2}+\left\|w_{a}-w\right\|_{L^{2}}^{2}+o_{a \nearrow a^{*}}(1), \\
\left\|\nabla w_{a}\right\|_{L^{2}}^{2} & =\|\nabla w\|_{L^{2}}^{2}+\left\|\nabla\left(w_{a}-w\right)\right\|_{L^{2}}^{2}+o_{a \nearrow a^{*}}(1) .
\end{aligned}
$$

Since $w_{a} \rightarrow w$ pointwise almost everywhere and $\left(w_{a}\right)_{a} \nearrow_{a^{*}}$ is bounded in $H^{1}$, the Brezis-Lieb's lemma (see e.g. [7]) implies that

$$
\left\|w_{a}\right\|_{L^{\frac{4}{d}+2}}^{\frac{4}{d}+2}=\|w\|_{L^{\frac{4}{d}+2}}^{\frac{4}{d}+2}+\left\|w_{a}-w\right\|_{L^{\frac{4}{d}+2}}^{\frac{4}{d}+2}+o_{a \nearrow a^{*}}(1) .
$$

It follows from (5.10), (5.7) and (5.13) that

$$
\frac{1}{2}\|\nabla w\|_{L^{2}}^{2}-\frac{1}{\frac{4}{d}+2}\|w\|_{L^{\frac{4}{d}+2}}^{\frac{4}{d}+2}+\frac{1}{2}\left\|\nabla\left(w_{a}-w\right)\right\|_{L^{2}}^{2}-\frac{1}{\frac{4}{d}+2}\left\|w_{a}-w\right\|_{L^{\frac{4}{d}+2}}^{\frac{4}{d}+2}=o_{a \nearrow a^{*}}(1) .
$$

Using the sharp Gagliardo-Nirenberg inequality and (5.9), we see that

$$
\frac{1}{2}\|\nabla w\|_{L^{2}}^{2}-\frac{1}{\frac{4}{d}+2}\|w\|_{L^{\frac{4}{d}+2}}^{\frac{4}{d}+2} \geq \frac{1}{2}\left(1-\left(\frac{\|w\|_{L^{2}}^{2}}{a^{*}}\right)^{\frac{2}{d}}\right)\|\nabla w\|_{L^{2}}^{2} \geq 0
$$

and similarly,

$$
\frac{1}{2}\left\|\nabla\left(w_{a}-w\right)\right\|_{L^{2}}^{2}-\frac{1}{\frac{4}{d}+2}\left\|w_{a}-w\right\|_{L^{\frac{4}{d}+2}}^{\frac{4}{d}+2} \geq \frac{1}{2}\left(1-\left(\frac{\left\|w_{a}-w\right\|_{L^{2}}^{2}}{a^{*}}\right)^{\frac{2}{d}}\right)\left\|\nabla\left(w_{a}-w\right)\right\|_{L^{2}}^{2} \geq 0 .
$$

We infer from the above inequalities, (5.14) and the fact $w \neq 0$ that

$$
\frac{1}{2}\|\nabla w\|_{L^{2}}^{2}-\frac{1}{\frac{4}{d}+2}\|w\|_{L^{\frac{4}{d}+2}}^{\frac{4}{d}+2}=0, \quad\|w\|_{L^{2}}^{2}=a^{*}, \quad \lim _{a \nearrow a^{*}}\left\|\nabla\left(w_{a}-w\right)\right\|_{L^{2}}^{2}=0 .
$$

This shows that $w$ is a non-negative optimizer for the sharp Gagliardo-Nirenberg inequality (1.19). Moreover, the later limit together with $w_{a} \rightarrow w$ weakly in $H^{1}$ imply that $w_{a} \rightarrow w$ strongly in $H^{1}$. Since $Q$ is the unique optimizer for the sharp Gagliardo-Nirenberg inequality up to translations and dialations (see e.g. [37]), we 
conclude that $w(x)=\beta_{0} Q\left(\lambda_{0} x-x_{0}\right)$ for some $\beta_{0}, \gamma_{0}>0$ and $x_{0} \in \mathbb{R}^{d}$. Since $\|w\|_{L^{2}}^{2}=a^{*}=\|Q\|_{L^{2}}^{2}$, we infer that $\beta_{0}=\lambda_{0}^{\frac{d}{2}}$, hence

$$
w(x)=\lambda_{0}^{\frac{d}{2}} Q\left(\lambda_{0} x-x_{0}\right)
$$

for some $\lambda_{0}>0$ and $x_{0} \in \mathbb{R}^{d}$. It remains to determine $\lambda_{0}$ and $x_{0}$ as follows.

We have from (5.8) that

$$
G\left(v_{a}\right)=\beta_{a}^{-\frac{\sigma}{2-\sigma}} G\left(w_{a}\right)
$$

and by the sharp Gagliardo-Nirenberg inequality,

$$
\begin{aligned}
\frac{1}{2}\left\|\nabla v_{a}\right\|_{L^{2}}^{2}-\frac{1}{\frac{4}{d}+2}\left\|v_{a}\right\|_{L^{\frac{4}{d}+2}}^{\frac{4}{d}+2} & \geq \frac{1}{2}\left(1-\left(\frac{a}{a^{*}}\right)^{\frac{2}{d}}\right)\left\|\nabla v_{a}\right\|_{L^{2}}^{2} \\
& =\frac{1}{2} \beta_{a}\left\|\nabla v_{a}\right\|_{L^{2}}^{2} \\
& =\frac{1}{2} \beta_{a}^{-\frac{\sigma}{2-\sigma}}\left\|\nabla w_{a}\right\|_{L^{2}}^{2} .
\end{aligned}
$$

It follows that

$$
\frac{I(a)}{a}=\frac{E\left(v_{a}\right)}{a} \geq \frac{\beta_{a}^{-\frac{\sigma}{2-\sigma}}}{a}\left(\frac{1}{2}\left\|\nabla w_{a}\right\|_{L^{2}}^{2}-\frac{1}{2} G\left(w_{a}\right)\right) .
$$

Since $w_{a} \rightarrow w$ strongly in $H^{1}$ and $G\left(w_{a}\right) \rightarrow G(w)$ as $a \nearrow a^{*}$, we see that

$$
\begin{aligned}
\beta_{a}^{\frac{\sigma}{2-\sigma}} \frac{I(a)}{a} & \geq \frac{1}{2}\left(\frac{\|\nabla w\|_{L^{2}}^{2}}{a^{*}}-\frac{G(w)}{a^{*}}\right)+o_{a \nearrow a^{*}}(1) \\
& =\frac{1}{2}\left(\frac{\lambda_{0}^{2}\|\nabla Q\|_{L^{2}}^{2}}{a^{*}}-\frac{\lambda_{0}^{\sigma} G\left(Q\left(\cdot-x_{0}\right)\right)}{a^{*}}\right)+o_{a \nearrow a^{*}}(1) \\
& =\frac{\lambda_{0}^{2} d}{4}-\frac{\lambda_{0}^{\sigma}}{2} G\left(Q_{0}\left(\cdot-x_{0}\right)\right)+o_{a \nearrow a^{*}}(1) .
\end{aligned}
$$

This implies that

$$
\liminf _{a \nearrow a^{*}} \beta_{a}^{\frac{\sigma}{2-\sigma}} \frac{I(a)}{a} \geq \frac{\lambda_{0}^{2} d}{4}-\frac{\lambda_{0}^{\sigma}}{2} G\left(Q_{0}\left(\cdot-x_{0}\right)\right) \geq \frac{\lambda_{0}^{2} d}{4}-\frac{\lambda_{0}^{\sigma}}{2} G\left(Q_{0}\right),
$$

where the last inequality follows from the Hardy-Littlewood rearrangement inequality and the fact $Q_{0}$ is radially symmetric and radially decreasing. Note that the equality holds if and only if $x_{0}=0$. We infer from (5.5) and (5.15) that $x_{0}=0$ and

$$
\lim _{a \nearrow a^{*}} \beta_{a}^{\frac{\sigma}{2-\sigma}} \frac{I(a)}{a}=\frac{\lambda_{0}^{2} d}{4}-\frac{\lambda_{0}^{\sigma}}{2} G\left(Q_{0}\right)=\min _{\lambda>0}\left(\frac{\lambda^{2} d}{4}-\frac{\lambda^{\sigma}}{2} G\left(Q_{0}\right)\right) .
$$

A direct computation shows that

$$
\lambda_{0}=\left(\frac{\sigma G\left(Q_{0}\right)}{d}\right)^{\frac{1}{2-\sigma}} .
$$

In conclusion, we have proved that up to a subsequence,

$$
\beta_{a}^{\frac{d}{2(2-\sigma)}} v_{a}\left(\beta_{a}^{\frac{1}{2-\sigma}} \cdot\right) \rightarrow \lambda_{0}^{\frac{d}{2}} Q\left(\lambda_{0} \cdot\right) \text { strongly in } H^{1} \text { as } a \nearrow a^{*}
$$

with $\lambda_{0}=\left(\frac{\sigma G\left(Q_{0}\right)}{d}\right)^{\frac{1}{2-\sigma}}$. Moreover, by the uniqueness of $Q$, we can conclude the above limit holds for the whole sequence $\left(v_{a}\right)_{a \nearrow a^{*}}$.

\section{APPENDIX}

The radial compact embedding. We first give a proof of the radial compact embedding (1.16). It is enough to show that if $\left(v_{n}\right)_{n \geq 1} \subset H_{\text {rd }}^{1}$ is such that $v_{n} \rightarrow 0$, then $v_{n} \rightarrow 0$ in $L^{q}$. Of course we can assume that $v_{n} \rightarrow 0$ in $L_{\text {loc }}^{q}$ and $v_{n} \rightarrow 0$ almost everywhere. It follows that

$$
\left\|v_{n}\right\|_{L^{q}}^{q}=\left\|v_{n}\right\|_{L^{q}(B)}^{q}+\left\|v_{n}\right\|_{L^{q}\left(B^{c}\right)}^{q}=\left\|v_{n}\right\|_{L^{q}\left(B^{c}\right)}^{q}+o_{n}(1)
$$


as $n \rightarrow \infty$, where $B=\left\{x \in \mathbb{R}^{d}:|x|<1\right\}$ and $B^{c}=\mathbb{R}^{d} \backslash B$. Since $\left(v_{n}\right)_{n \geq 1}$ is bounded in $H^{1}$, by (1.15), there exists $C(d)>0$ independent of $n$ such that

$$
\left|v_{n}(x)\right|^{q} \leq C(d)|x|^{-\frac{d q}{2}} .
$$

The last term is integrable on $B^{c}$ since $\frac{d q}{2}>d$. By the dominated convergence,

$$
\left\|v_{n}\right\|_{L^{q}\left(B^{c}\right)}^{q} \rightarrow 0
$$

as $n \rightarrow \infty$.

The uniqueness of positive radial solution for (1.7). Using the general results of Shioji-Watanabe [35], we have that for any $\omega>-\mu_{1}, 0<\sigma<1, d \geq 3$ and $0<\alpha<\frac{4}{d-2}$, there exists a unique positive solution to (1.7). In fact, in [35, Theorem 1], Shioji-Watanabe proved a uniqueness result for

$$
\phi^{\prime \prime}(r)+\frac{f^{\prime}(r)}{f(r)} \phi^{\prime}(r)+g(r) \phi(r)+h(r) \phi^{p}(r)=0, \quad r \in(0,+\infty)
$$

under appropriate assumptions on $f(r), g(r)$ and $h(r)$. In our case, we have

$$
f(r)=r^{d-1}, \quad g(r)=c r^{-\sigma}-\omega, \quad h(r)=1, \quad p=\alpha+1 .
$$

Applying Theorem 1 in [35], the uniqueness of positive solution to (1.7) holds if the following conditions are satisfied:

(I) $g \in C^{1}(0,+\infty), h \in C^{3}(0,+\infty)$ and $h(r)>0$ for every $r \in(0,+\infty)$.

(II)

$$
\lim _{r \rightarrow 0} r^{1-d} \int_{0}^{r} \tau^{d-1}(|g(\tau)|+h(\tau)) d \tau=0
$$

(III) There exists $r_{0} \in(0,+\infty)$ such that

(i) $r^{d-1} g(r), r^{d-1} h(r) \in L^{1}\left(0, r_{0}\right)$;

(ii)

$$
r^{d-1}(|g(r)|+h(r))\left(\frac{r_{0}^{2-d}-r^{2-d}}{2-d}\right) \in L^{1}\left(0, r_{0}\right)
$$

(IV) $\lim _{r \rightarrow 0} a(r)<\infty, \lim _{r \rightarrow 0}|b(r)|<\infty, \lim _{r \rightarrow 0} c(r) \in[0, \infty], \lim _{r \rightarrow 0} a(r) g(r)=0$ and $\lim _{r \rightarrow 0} a(r) h(r)=$ 0 , where

$$
\begin{aligned}
& a(r)=r^{\frac{2(d-1)(p+1)}{p+3}} h(r)^{-\frac{2}{p+3},} \\
& b(r)=-\frac{1}{2} a^{\prime}(r)+\frac{d-1}{r} a(r), \\
& c(r)=-b^{\prime}(r)+\frac{d-1}{r} b(r) .
\end{aligned}
$$

(V) There exists $r_{1} \in(0, \infty)$ such that $G(r)>0$ on $\left(0, r_{1}\right)$ and $G(r)<0$ on $\left(r_{1},+\infty\right)$, where

$$
G(r)=-b(r) g(r)+\frac{1}{2} c^{\prime}(r)+\frac{1}{2}\left(a^{\prime}(r) g(r)+a(r) g^{\prime}(r)\right) .
$$

(VI) $G^{-} \neq 0$, where $G^{-}:=\min \{G(r), 0\}$ for $r \in(0,+\infty)$.

Under the assumptions $\omega>-\mu_{1}, 0<\sigma<1, d \geq 3$ and $0<\alpha<\frac{4}{d-2}$, it is easy to see that (I), (II) and (III) hold. By a direct computation, we have

$$
\begin{aligned}
a(r) & =r^{\frac{2(d-1)(\alpha+2)}{\alpha+4}}, \\
b(r) & =\frac{2(d-1)}{\alpha+4} r^{\frac{(2 d-3) \alpha+4(d-2)}{\alpha+4}}, \\
c(r) & =\frac{2(d-1)(4-(d-2) \alpha)}{(\alpha+4)^{2}} r^{\frac{2(d-2) \alpha+4(d-3)}{\alpha+4}} .
\end{aligned}
$$

Since $d \geq 3$ and $0<\sigma<1$, we see that (IV) is satisfied. Finally, we have

$$
\begin{aligned}
G(r) & =A r^{\frac{(2 d-3) \alpha+4(d-2)}{\alpha+4}}+B r^{\frac{(2 d-3) \alpha+4(d-2)}{\alpha+4}-\sigma}+C r^{\frac{(2 d-3) \alpha+4(d-2)}{\alpha+4}-2} \\
& =\left(A r^{2}+B r^{2-\sigma}+C\right) r^{\frac{(2 d-3) \alpha+4(d-2)}{\alpha+4}-2}
\end{aligned}
$$


where

$$
\begin{aligned}
& A=-\frac{\omega \alpha(d-1)}{\alpha+4}, \\
& B=\frac{c((2 d-2-\sigma) \alpha-4 \sigma)}{2(\alpha+4)}, \\
& C=\frac{(d-1)(4-(d-2) \alpha)[2(d-2) \alpha+4(d-3)]}{(\alpha+4)^{3}} .
\end{aligned}
$$

Since $A<0$ and $C>0$, there exists $r_{1}>0$ such that $G(r)>0$ on $\left(0, r_{1}\right)$ and $G(r)<0$ on $\left(r_{1}, \infty\right)$ which shows (V) and aslo (VI).

\section{ACKNOWLEDGEMENT}

This work was supported in part by the Labex CEMPI (ANR-11-LABX-0007-01). The author would like to express his deep gratitude to his wife - Uyen Cong for her encouragement and support. He also would like to thank the reviewer for his/her helpful comments and suggestions.

\section{REFERENCES}

[1] A. H. Ardila and V. D. Dinh, Some qualitative studies of the focusing inhomogeneous Gross-Pitaevskii equation, preprint arXiv: $1903.04644,2019.3$

[2] J. Bellazzini, N. Boussaid, L. Jeanjean and N. Visciglia, Existence and stability of standing waves for supercritical NLS with a partial confinement, Comm. Math. Phys. 353(1):229-339, 2017. 6, 7

[3] R. Benguria and L. Jeanneret, Existence and uniqueness of positive solutions of semilinear elliptic equations with coulomb potentials on $\mathbb{R}^{3}$, Commun. Math. Phys. 104:291-306, 1986. 1

[4] A. Bensouilah, V. D. Dinh and S. Zhu, On stability and instability of standing waves for the nonlinear Schrödinger equation with an inverse-square potential, J. Math. Phys. 59:101505, 2018. 1

[5] H. Berestycki and P. L. Lions, Nonlinear scalar field equations, I: Existence of ground state, Arch. Ration. Mech. Anal. 82:313-345, 1983. 5, 20

[6] N. Burq, F. Planchon, J. Stalker and A. S. Tahvildar-Zadeh, Strichartz estimates for the wave and Schrödinger equations with inverse-square pontential, J. Funct. Anal. 203(2):519-549, 2003. 1

[7] H. Brezis and E. Lieb, A relation between pointwise convergence of functions and convergence of functionals, Proc. Amer. Math. Soc. 88:486-490, 1983. 5, 20

[8] J. M. Chadam and R. T. Glassey, Global existence of solutions to the Cauchy problem for time-dependent Hartree equations, J. Math. Phys. 16:1122-1130, 1975. 1

[9] E. Csobo and F. Genoud, Minimal mass blow-up solutions for the $L^{2}$ critical NLS with inverse-square potential, Nonlinear Anal. 168:110-129, 2018. 1

[10] C. R. de Oliveira, Intermediate spectral theory and quantum dynamics, Progress in Mathematical Physics 54, Birkhäuser, Berlin, 2009. 2

[11] V. D. Dinh, Global existence and blowup for a class of the focusing nonlinear Schrödinger equation with inverse-square potential, J. Math. Anal. Appl. 468:270-303, 2018. 1

[12] V. D. Dinh, On instability of radial standing waves for the nonlinear Schrödinger equation with inverse-square potential, preprint arXiv.1806.01068, 2018. 10

[13] V. D. Dinh, On nonlinear Schrödinger equations with repulsive inverse-power potentials, preprint arXiv: $1812.08405,2018$. 1,13

[14] N. Fukaya and M. Ohta, Strong instability of standing waves for nonlinear Schrödinger equations with attractive inverse power potential, preprint arXiv:1804.02127, 2018. 1, 3, 7, 8, 10

[15] R. Fukuizumi and M. Ohta, Instability of standing waves for nonlinear Schrödinger equations with potentials, Differential Integral Equations 16(6):691-706, 2003. 8, 14

[16] R. T. Glassey, On the blowing up of solutions to the Cauchy problem for nonlinear Schrödinger equations, J. Math. Phys., 18:1794-1797, 1977. 3, 12

[17] D. Gilbarg and N. Trudinger, Elliptic partial differential equations of second order, Third Edition, Springer-Verlag, Berlin, 2001. 2

[18] Y. Guo and R. Seiringer, On the mass concentration for Bose-Einstein condensates with attractive interactions, Lett. Math. Phys. 104:141-156, 2014. 6

[19] Y. J. Guo, X. Y. Zeng and H. S. Zou, Energy estimates and symmetry breaking in attractive Bose-Einstein condensates with ring-shaped potentials, Ann. Inst. Henri Poincaré Nonlinear Anal. 33:809-828, 2016. 6

[20] Q. Guo, H. Wang and X. Yao, Dynamics of the focusing 3D cubic NLS with slowly decaying potential, preprint arXiv:1811.37578, 2018. 1

[21] H. Hajaiej, Cases of equality and strict inequality in the extended Hardy-Littlewood inequalities, Proc. Roy. Soc. Edinburgh Sect. A 135(3):643-661, 2005. 16

[22] N. Hayashi and T. Ozawa, Time decay of solutions to the Cauchy problem for time-dependent Schrödinger-Hartree equations, Commun. Math. Phys. 110:467-478, 1987. 1 
[23] R. Killip, C. Miao, M. Visan, J. Zhang and J. Zheng, The energy-critical NLS with inverse-square potential, Discrete Contin. Dyn. Syst. 37:3831-3866, 2017. 1

[24] R. Killip, J. Murphy, M. Visan and J. Zheng, The focusing cubic NLS with inverse-square potential in three space dimension, Differential Integral Equations 30(3-4):161-206, 2017. 1

[25] R. Killip, C. Miao, M. Visan, J. Zhang and J. Zheng, Sobolev spaces adapted to the Schrödinger operator with inverse-square potential, Math. Z. 288(3-4):1273-1298, 2018. 1

[26] E. H. Lieb and M. Loss, Analysis, Second edition, Graduate Studies in Mathematics 14, AMS, Providence, 2001. 8, 20

[27] X. Li and J. Zhao, Orbital stability of standing waves for Schrödinger type equations with slowly decaying linear potential, Comput. Math. Appl. 2019 (in press). 1, 5

[28] P. L. Lions, Some remarks on Hartree equation, Nonlinear Anal. 5(11):1245-1256, 1981. 1

[29] P. L. Lions, The concentration-compactness principle in the calculus of variations. The locally compact case: Part 1 , Ann. Inst. Henri Poincaré 1:109-145, 1984. 5

[30] J. Lu, C. Miao and J. Murphy, Scattering in $H^{1}$ for the intercritical NLS with an inverse-square potential, J. Differential Equations 264(5):3174-3211, 2018. 1

[31] C. Miao, J. Zhang and J. Zheng, Nonlinear Schrödinger equation with coulomb potential, preprint arXiv:1809.06685, 2018. 1

[32] H. Mizutani, Strichartz estimates for Schrödinger equations with slowly decaying potentials, preprint arXiv:1808.06987, 2018. 1

[33] N. Okazawa, T. Suzuki and T. Yokota, Energy methods for abstract nonlinear Schrödinger equations, Evol. Equ. Control Theory 1:337-354, 2012. 1

[34] T. V. Phan, Blow-up profile of Bose-Einstein condensate with singular potentials, J. Math. Phys. 58:072301, 2017. 6

[35] N. Shioji and K. Watanabe, A generalized Pohozaev identity and uniqueness of positive radial solutions of $\Delta u+g(r) u+$ $h(r) u^{p}=0$, J. Differential Equations 255:4448-4475, 2013. 3, 22

[36] Q. Wang and D. Zhao, Existence and mass concentration of 2D attractive Bose-Einstein condenstates with periodic potentials, J. Differential Equations 262:2684-2704, 2017. 6

[37] M. I. Weinstein, Nonlinear Schrödinger equations and sharp interpolation estimates, Commun. Math. Phys. 87:567-576, 1983. 20

[38] X. Zhang, On the Cauchy problem of 3-D energy-critical Schrödinger equations with subcritical perturbations, J. Differential Equations 230:422-445, 2006. 1

[39] J. Zhang and J. Zheng, Scattering theory for nonlinear Schrödinger equation with inverse-square potential, J. Funct. Anal. 267:2907-2932, 2014. 1, 7

[40] J. Zheng, Focusing NLS with inverse square potential, J. Math. Phys. 59:111502, 2018. 1

(V. D. Dinh) Laboratoire Paul Painlevé UMR 8524, Université de Lille CNRS, 59655 Villeneuve D’Asce Cedex, France and Department of Mathematics, HCMC University of Pedagogy, 280 An Duong Vuong, Ho Chi Minh, VIETNAM

E-mail address: contact@duongdinh.com 